\title{
2 Funktionsverbgefüge: Abgrenzungsversuche in der Forschung
}

\subsection{Einleitung}

Die Klage über die Vagheit des Begriffs ,Funktionsverbgefüge“ (im Weiteren: FVG) ist längst zu einem Topos geworden. So hatte bereits von Polenz (1987: 169) festgestellt, dass hier ,durch terminologische Verallgemeinerung Verwirrung gestiftet worden“ sei, Tao (1997: 7) beklagt die „Vielfalt der Auffassungen darüber, was ein FVG ist“, und noch in der jüngeren Literatur wird das „Fehlen überzeugender und allgemein akzeptierter Abgrenzungskriterien“ konstatiert (Seifert 2004: 2, vgl. auch van Pottelberge 2001: 2; 2007: 437; Winhart 2005: 1; Heine 2006: 17; Storrer 2006: 149f.; Kamber 2008: 9). ${ }^{1}$

Die immer wieder thematisierten Schwierigkeiten bei der Definition eines sprachwissenschaftlichen Begriffs FVG dürften zunächst wissenschaftsgeschichtliche Gründe haben: Substantivische Prädikatsausdrücke wie zur Entscheidung kommen, Anwendung finden, Hilfe leisten, denen stammgleiche Simplizia entscheiden, angewandt werden, helfen entsprechen, hat die Sprachwissenschaft nicht selbst als Gegenstand entdeckt. Vielmehr war das Thema bereits durch die Sprachkritik vorgegeben, die diese Erscheinungen als „Sprachschwulst“, „Verbzerstörung“, „Hauptwörterkrankheit“ und letztlich als Symptom eines allgemeinen „Sprachverfalls“ in der technisierten Welt getadelt hat. ${ }^{2}$ Die wissenschaftliche Beschäftigung mit Nomen-Verb-Gefügen dieser Art, die mit den Untersuchungen von Kolb (1962), von Polenz (1963) und Daniels (1963) einsetzt, ist eine unmittelbare Reaktion auf diese als einseitig und unbegründet empfundenen Verurteilungen. ${ }^{3}$ Wie eng die sprachwissenschaftliche Begriffsbildung zunächst auf die Sprachkritik - und damit auf eine Sprachbetrachtung, die

1 In diesem Sinne äußern sich ferner z. B. auch Persson (1992: 153); Eisenberg (2006a: 309f.); Elsayed (2000: 1); Fleischer (2001: 117).

2 Eine Zusammenstellung solcher Urteile bietet Daniels (1963: 9f.); vgl. auch Braun (1993: 231236); von Polenz (1999: 351f.).

3 Auffallend ist dabei in wissenschaftsgeschichtlicher Hinsicht, dass die frühen 60er Jahre generell von einer lebhaften Debatte zwischen Sprachwissenschaftlern und Sprachkritikern gekennzeichnet sind, die sich vor allem an dem antifaschistischen „Wörterbuchs des Unmenschen“ von Sternberger/Storz/Süskind (1968) sowie Karl Korns „Sprache in der verwalteten Welt“ (1962) entzündete, vgl. dazu von Polenz (1999: 317f.).

Ә Open Access. (C) 2021 Volker Harm, publiziert von De Gruyter. (c) BY Dieses Werk ist lizenziert unter der Creative Commons Attribution-NonCommercial-NoDerivatives 4.0 Lizenz.

https://doi.org/10.1515/9783110661255-002 
letztlich als vorwissenschaftlich gelten muss - bezogen war, belegen nicht zuletzt die Begriffe „Funktionsverbum“ (zuerst Kolb 1962: 381, vgl. von Polenz 1963: 12) und „Funktionsverbgefüge“ (zuerst Engelen 1968: 289) selbst, bringen diese doch programmatisch zum Ausdruck, dass solche Fügungen aufgrund einer wie auch immer zu bestimmenden - Funktionalität eben nicht überflüssig seien. Der Terminus ,Funktionsverbgefüge ' ist damit gewissermaßen ein Kampfbegriff, der im Zuge der Auseinandersetzung zwischen Sprachwissenschaft und Sprachkritik geprägt wurde. Das vorwissenschaftliche Erbe, das diesem Begriff anhaftet, mag einer der Gründe für dessen notorische Unschärfe sein.

Im Folgenden sollen die maßgeblichen Definitionsversuche für FVG vorgestellt und diskutiert werden. Dies geschieht allerdings nicht in Form eines chronologisch angelegten Gangs durch die Geschichte der FVG-Abgrenzungen - ein solches Unterfangen würde angesichts der Fülle der zum Thema vorgelegten Literatur schnell labyrinthische Züge annehmen. ${ }^{4}$ Stattdessen wird hier versucht, in einer thematisch gegliederten Übersicht gewissermaßen die Brennpunkte des Definitionsproblems zu erfassen. Einer dieser Brennpunkte - und wohl der zentrale - ist die Frage nach dem „semantischen Mehrwert“ (von Polenz 1963: 13) der betreffenden Fügungen gegenüber den jeweiligen einfachen Verben. Ein weiterer Problembereich betrifft die syntaktische Struktur von FVG: Wie ist die verbale, wie die nominale Komponente beschaffen und durch welche syntaktischen Strukturen sind FVG definierbar? Zu klären ist schließlich, ob es operationalisierbare Testverfahren für die Ermittlung von FVG gibt.

\subsection{Der ,semantische Mehrwert` der FVG}

\subsubsection{Aktionsartenmarkierung}

Da in den 1960er Jahren die Verteidigung der FVG gegen die Sprachkritik das vordringlichste Anliegen der Forschung war, konzentrierte man sich vor allem darauf, die spezifischen Leistungen der FVG im Verhältnis zum einfachen Verb herauszuarbeiten. ${ }^{5}$ Ganz dem Ziel, den ,semantischen Mehrwert ${ }^{\star}$ der von der

4 Zur Geschichte der FVG-Forschung liegen zudem bereits einige Darstellungen vor, z. B. Bahr (1977: 13-18), Guttmacher (1980: 13-45), Yuan (1986, 22-72), Böhmer (1994) sowie besonders die ausführliche Auseinandersetzung in van Pottelberge (2001: 15-253).

5 Wenn im folgenden Forschungsüberblick durchgehend von „Funktionsverbgefügen“ die Rede ist, so ist die Verwendung dieses Begriffes teilweise ahistorisch: von Polenz (1963) spricht noch von „Funktionsverbformeln“, Heringer (1968) von „Funktionsverbfügungen“, Daniels (1963) lediglich von „nominalen Umschreibungen“. Der Terminus „Funktionsverbgefüge“ 
Stilkritik beanstandeten Erscheinungen nachzuweisen, ist auch die bereits genannte Untersuchung von Polenz' (1963) gewidmet. ${ }^{6}$ Dieses ausgeprägte Rechtfertigungsbestreben lässt von Polenz’ Definition von „Funktionsverb“ deutlich erkennen:

Wir können sie [die Verben der behandelten Gefüge, V. H.] ,Funktionsverben` nennen, weil sie das Verbalsubstantiv funktionell in das Vorgangsgefüge einfügen, indem sie die syntaktische Funktion des im Verbalsubstantiv aufgehobenen Grundverbums übernehmen, während dessen Sachkern im Nennglied [d. h. im Substantiv, V. H.] wirksam bleibt. Diese analytische Aufspaltung des Vorgangsbegriffes in Funktion und Inhalt ermöglicht zugleich eine Modifizierung des Vorgangsbegriffes auf der funktionellen Seite. Die Funktionsverben übernehmen also nicht nur die Funktion des Grundverbs, sondern verändern sie auch. Die besondere Leistung der Funktionsverben besteht also nicht in ,bloßer' Funktion; sie zeigt sich mehr oder weniger auch in Funktionsnuancen, deren das Grundverb nicht fähig.

(von Polenz 1963: 26f.; Hervorhebungen im Original)

Die angesprochenen Funktionsnuancen betreffen im Wesentlichen die „zeitphasenhafte Vorgangsabstufung“ (von Polenz 1963: 27), eine Erscheinung, die in der Sprachwissenschaft herkömmlicherweise als ,Aktionsart' bezeichnet wird. ${ }^{7}$ von Polenz (1963: 14-22) unterscheidet folgende Aktionsarten, die durch FVG ausgedrückt werden können:

- konklusive Aktionsart, die die dem Handlungsabschluss vorausliegende Phase in den Vordergrund stellt (zum Abschluss, zum Einsturz, zur Explosion, zum Spalten kommen, bringen $)^{8}$

wurde zuerst von Engelen (1968) verwendet und hat sich seitdem durchgesetzt. Um Begriffsverwirrung zu vermeiden, wird hier, auch für die Literatur vor 1968, konsequent von „Funktionsverbgefüge“ (FVG) gesprochen.

6 Ausdrücklich von einem „semantischen Mehrwert“ spricht von Polenz (1963: 13); vgl. noch von Polenz (1999: 352); Rösch (1990: 180); Seifert (2004: 72).

7 Zum Begriff ,Aktionsart' und seiner Anwendung auf das Deutsche Heidolph/Flämig/Motsch (1984: 501): „Unter der Aktionsart des Verbs [...] versteht man die sprachliche Kennzeichnung der Art und Weise eines Vorgangs, besonders die objektive Darstellung der Phasen eines Geschehens, z. B. ,Einsetzen“ (erblühen), ,Verlauf‘ (blühen), ,Enden` (verblühen).“ - Zuweilen, etwa bei Schmidt (1968: 36-45), Helbig (1983: 178), Wolf (1987: 222) und Tao (1997: 74f.), wird auch das Kausativum als ,Aktionsart ' bezeichnet. Da beim Kausativum jedoch keine Phasenabstufung wie beim Inchoativ oder Durativ vorliegt, sollte das Kausativum nicht als ,Aktionsart' klassifiziert werden, vgl. auch van Pottelberge (2001: 163).

8 Zum Begriff ,konklusive Aktionsart‘ vgl. auch Di Meola (1994: 167). In jüngeren Arbeiten, z. B. von Polenz (1987: 173f.) sowie Helbig (1984: 178f.), wird auf die konklusive Aktionsart nicht eingegangen, stattdessen wird übergreifend von inchoativer Aktionsart gesprochen. 
- inchoative Aktionsart, die den Beginn eines Geschehens bezeichnet (in Gebrauch nehmen, in Betracht ziehen, zur Kenntnis nehmen)

- progressive Aktionsart, die das Andauern eines Geschehen oder Zustands bezeichnet (in Verhandlung, in Frage, in Beziehung, in Verbindung stehen)

von Polenz bemüht sich im Einzelnen nachzuweisen, worin der Mehrwert dieser FVG gegenüber entsprechenden einfachen Verben besteht: So bezeichne konklusives zur Entscheidung bringen im Gegensatz zu punktuellem entscheiden „eine resultatbezogene Tätigkeit“ (1963: 15). Inchoatives in Erwägung, in Betracht ziehen beinhalte gegenüber erwägen, betrachten „eine bewußte Verzögerung des Vorgangs“ und drücke damit „eine bedächtige, vorsichtige Art des ,Betrachtens“ oder ,Erwägens““ aus (1963: 19). Die progressiven FVG des Typs in Verhandlung stehen werden in besonderer Weise gewürdigt: Mit ihrer „Verbindung von Ruhe und potentieller Bewegung“ seien sie „das Äußerste, was die neuen Funktionsverben für die Vorgangsverzögerung und -abstufung leisten können“ (1963: 21).

In der Aktionsartenmarkierung durch FVG hat von Polenz sogar Züge eines analytischen Sprachbaus erkennen wollen, der im Deutschen der Gegenwart sichtbar werde. FV werden damit quasi zu Hilfsverben der Aktionsartenmarkierung, die anstelle der angestammten Aktionsartenmarkierung durch Partikelverben (wie in anbraten, losrennen) treten:

Gerade hier [bei FVG, nicht mehr nur bei der Wortbildung, V. H.] hat die Aktionsartenforschung zum heutigen Deutsch einzusetzen. Wenn es im Deutschen moderne Ansätze zur Bezeichnung von Aktionsarten gibt, dann sind sie vor allem in dem analytischen Sprachbaumittel der Umschreibung mit Hilfs- und Funktionsverben zu suchen.

(von Polenz 1963: 28)

Offenbar hatte aber auch schon von Polenz (1963) bemerkt, dass eine Vielzahl zeitgenössischer Belege für FVG keine aktionale Differenzierung in dem von ihm postulierten Sinne enthalten. Dieses empirischen Problems entledigt sich von Polenz, indem er solche Verwendungen als Verirrungen der Sprecher abqualifiziert:

9 Die Auffassung, FV seien grammatische Wörter zur Kennzeichnung von Aktionsarten, hat später vor allem Steinitz (1981: 84f.) vertreten, hier in Bezug auf kommen. 
Leider werden sie [FVG, V. H.] heute im sprachlichen Massenverbrauch des öffentlichen Lebens derart unbedacht und unkontrolliert verwendet, daß es dem normalen Sprachteilhaber kaum noch möglich ist, die wirklichen Bedeutungsnuancen von den bloßen Wucherungen zu unterscheiden.

(von Polenz 1963: 29f.)

Die Erkenntnis, dass FVG durchaus nicht immer einen aktionalen Unterschied gegenüber dem einfachen Verb ausdrücken, hat sich zwar schon früh durchgesetzt (vgl. Heringer 1968: 95f. zu in Wegfall kommen vs. wegfallen); sie hat freilich nicht dazu geführt, dass die grundlegende Funktion der Aktionsartenmarkierung für die Definition der Klasse FVG grundsätzlich in Frage gestellt wurde.

Bedenken gegenüber der lange als selbstverständlich akzeptierten Hypothese, dass die primäre Funktion von FVG in der Aktionsartenmarkierung bestehe, sind in aller Deutlichkeit zuerst durch van Pottelberge (2001) erhoben worden. van Pottelberge (2001: 213-219) demonstriert anhand einiger Beispiele, dass in Bezug auf die Aktionsart kein Unterschied zwischen dem FVG und dem entsprechenden einfachen Verb bestehe. So sei nicht erkennbar, inwiefern sich etwa zur Verfügung stehen in (1a) - laut von Polenz (1987: 174) und Helbig/Buscha (2001: 81) ein FVG - in aktionaler Hinsicht von verfügen in (1b) unterscheiden solle:

a. Die Verantwortung dafür [...], daß ihr zwar die Richtung mitgeteilt wird, in die sie sich bewegen soll, nicht aber, welche Mittel zur Verfügung stehen, trägt die Regierung (Beleg aus FAZ 06.04.1994, 1; zit. in van Pottelberge 2001: 215).

b. Seine Enthüllungen machten lediglich erkennbar, daß auch er nicht über Wundermittel verfügt (Beleg aus FAZ 23.06.1993, 1; ebd.).

In diesem Sinne ließen sich viele weitere traditionellerweise als FVG klassifizierte Verbindungen anführen, bei denen hinsichtlich der Aktionsart kein Unterschied zwischen der Nomen-Verb-Fügung und einem entsprechenden Verb erkennbar ist. Dies betrifft insbesondere eine große Zahl von Verbindungen mit kommen, welches seit von Polenz als Ausdruck einer inchoativen Aktionsart gilt und zum Kernbestand der FV gerechnet wird:

(2) zur Geltung kommen, zur Darstellung kommen, zur Aufführung kommen, zur Anwendung kommen, zum Ausdruck kommen, zur Verteilung kommen 
Läge hier inchoative Bedeutung vor, müssten die Fügungen in (2) mit beginnen paraphrasierbar sein (wie losrennen mit beginnen zu rennen). Dies ist indes nicht der Fall: Das Stück kommt zur Aufführung ist z. B. nicht gleichzusetzen mit Die Aufführung des Stücks beginnt, sondern bedeutet schlicht ,das Stück wird aufgeführt', ist also als passivisch aufzufassen (vgl. Seifert 2004: 91). Bei einer großen Gruppe von Fügungen, die herkömmlicherweise zu den FVG gerechnet werden, liegt somit keine inchoative Bedeutung vor. Dies hat die jüngere Forschung allerdings zum Teil dazu verleitet, schlicht zwei Arten von FVG anzunehmen: die gewissermaßen echten FVG mit Aktionsartendifferenzierung sowie eine Klasse von „,indifferenten FVG“ (Tao 1997: 77; vgl. bereits Heringer 1968: 94f.). Nach welchem Kriterium diese ,indifferenten“ Fügungen trotzdem zu den FVG gerechnet werden sollen, bleibt dabei unklar. In der rezenteren Forschung, hier vor allem etwa bei Eroms (2000: 167), Helbig/Buscha (2001: 92f.) sowie bei Seifert (2004: 72), wird die Aktionsartenmarkierung allenfalls noch als eine Funktion von FVG unter vielen anderen gesehen, durch die sich diese Klasse auszeichnet.

\subsubsection{Kausativa}

Als eine besondere Leistung der von der Sprachkritik bekämpften „Streckformen“ hat Kolb (1962) neben der Aktionsartenmarkierung auch die Bildung von Kausativa herausgestellt. Da die synthetischen Kausativierungen des Typus trinken - tränken, sitzen - setzen usw. im Neuhochdeutschen nicht mehr produktiv seien, so Kolbs Argumentation, stehe mit den aus Präp. + nomen actionis + bringen gebildeten Verbindungen ein Ausdrucksmittel zur Verfügung, das diese durch die Sprachgeschichte gerissene Lücke auffüllen könne: „Die neuen Bildungen sind in der Sprache notwendig geworden, seitdem die alten Kausativa ihren Sinn und ihre Funktion verloren hatten“ (1962: 380). ${ }^{10}$ In einigen Fällen (zur Vorlage bringen, in Abzug bringen) erkennt Kolb allerdings keine „gegenüber dem Verbum zusätzliche Funktion“ (1962: 383); die Kausativbildungen hätten daher ihren „historisch gerechtfertigten Bereich“ verlassen und seien - hier wird ausdrücklich der Sprachkritik recht gegeben - als „Wucherungen“ (ebd.) anzusehen.

10 Einen Zusammenhang zwischen dem Aufkommen von FVG und dem Produktivitätsverlust der jan-, ôn-, ên-Bildungen sehen auch von Polenz (1963: 16), Relleke (1974: 23) sowie Tao (1997: 78f.). Den empirischen Nachweis eines solchen sprachhistorischen Zusammenhangs bleiben aber auch diese Untersuchungen schuldig. 
Auch von Polenz (1963) weist auf die Kausativumschreibung hin, die mit FVG möglich sei. Eine Kausativumschreibung liegt nach von Polenz z. B. vor bei zum Kochen bringen, zum Lachen bringen, zur Sprache/zum Sprechen bringen, in Bewegung bringen, aber auch bei Verbindungen mit setzen: in Bewegung, in Umlauf, in Gang setzen. Das Besondere der Konstruktion mit bringen gegenüber den angestammten Umschreibungen mit lassen oder bewirken sieht von Polenz darin, dass der Konstruktion mit lassen/bewirken „die zeitliche ,Verumständlichung“, das vorbereitende Hinführen auf den eigentlichen Vorgang, das bringen mit seiner Richtungspräposition ausdrücken kann“, fehle (von Polenz 1963: 16). (Ob diese Leistung neben den bringen-Konstruktionen auch für die Fügungen mit setzen gilt, die von Polenz ja ausdrücklich zu den kausativen FVG zählt, wird dabei offen gelassen.)

In der breiten Auseinandersetzung mit FVG seit den späten sechziger Jahren wurden die Möglichkeiten der Kausativbildung durch FVG dann besonders intensiv diskutiert (vgl. Heringer 1968; Herrlitz 1973; Persson 1975; 1992, Esau 1976; von Polenz 1987). Die Forschung hat sich dabei unter anderem um eine genauere prädikatenlogische Charakterisierung des bei Kolb (1962) und von Polenz (1963) noch weitgehend intuitiv verwendeten Begriffs ,kausativ‘ bemüht. So beschreibt von Polenz (1987) im Anschluss an Vorarbeiten von Heringer (1968: 83f.) und Persson (1975: 148) die Struktur kausativer FVG als eine Art Prädikatskomplex, bei dem

in die 2. Argumentstelle einer Oberprädikation mit dem KAUSATIV-Prädikat BEWIRKEN über eine Aktionsart A eine Prädikation P eingebettet ist. BEWIRKEN wird im FVG durch FV wie bringen, setzen, versetzen ausgedrückt, $\mathrm{P}$ durch das $\mathrm{N}$ [omen] $\mathrm{A}$ [ctionis]. So ist z. B. die Prädikation ,y bewegt sich' über die implizite Aktionsart ,es kommt dazu, daß ...' in die Ober-Prädikation , $\mathrm{x}$ bewirkt, daß ...` eingebettet, im FVG ausgedrückt als $x$ bringt/setzt/versetzt $y$ in Bewegung.

(von Polenz 1987: 172) ${ }^{11}$

P ist dabei entweder Handlung, Tätigkeit, Vorgang, Ereignis oder Zustand mit einer Argumentstelle y, „die in der Regel nicht mit x identisch ist“ (von Polenz 1987: 173). Die Aktionsart A, die mit $P$ verbunden ist, ist inchoativ, bezeichnet somit den Beginn oder das Zustandekommen einer Handlung, eines Vorgangs usw. Kausative FVG vom Typ in Bewegung bringen implizieren daher gewissermaßen

11 Vgl. auch Persson (1992: 154) und Esau (1976: 151). 
ein entsprechendes inchoatives FVG mit kommen: in Bewegung bringen ist ,bewirken, dass etwas in Bewegung kommt' . Die prädikatenlogische Formel für eine kausative Struktur dieses Typs lautet daher nach von Polenz:

$$
\operatorname{BEWIRK}\left(\mathrm{x}_{\mathrm{ag}}, \mathrm{A}(\mathrm{P}(\mathrm{y} \ldots))\right)
$$

(von Polenz 1987: 173)

Dass Konstruktionen wie in Bewegung bringen, zum Lachen bringen sich im Rahmen der bei von Polenz postulierten logischen Struktur als Kausativa erfassen lassen, ist allgemein akzeptiert. ${ }^{12}$ Problematisch hingegen sind Fügungen wie in (4), die eigentlich seit jeher zum Kernbestand der FVG gehört haben.

(4) zum Ausdruck bringen, zur Aufführung bringen, zur Darstellung bringen, zur Verteilung bringen, zur Anwendung bringen

von Polenz (1987: 184) macht zurecht darauf aufmerksam, dass hier Referenzidentität zwischen dem Agens von BEWIRKEN und dem Agens der BEWIRKTEN HANDLUNG $(\mathrm{P})$ besteht: , $\mathrm{x}$ bewirkt, dass es dazu kommt, dass $\mathrm{P}(\mathrm{x} \text {...) })^{\star}$. Das heißt z. B. für zum Ausdruck bringen: , $\mathrm{x}$ bewirkt, dass es dazu kommt, dass $\mathrm{x}$ etwas ausdrückt‘. Da man eigenes Tun nicht bewirken könne, bezeichnet von Polenz Fügungen dieser Art als „pseudokausativ“ oder „reflexiv-kausativ“. Kausativa im Sinne der semantischen Struktur in (3) sind dagegen Verbindungen, deren $\mathrm{x}$ - und $\mathrm{y}$-Argumente jeweils nicht übereinstimmen, bei denen also ein $\mathrm{x}$ bewirkt, dass ein y etwas tut oder mit diesem y etwas geschieht. Dies ist z. B. bei den Fügungen in (5) der Fall .

(5) a. zum Laufen bringen, zum Lachen bringen, zum Schreiben bringen, zum Stehen bringen

b. in Bewegung setzen, in Gang setzen

Gegenüber den genuinen Kausativa in (5) sind die Pseudokausativa in (4) laut von Polenz (1987: 184) „semantisch künstlich und überflüssig“. Sie weisen gegenüber den entsprechenden einfachen Verben gerade nicht den für FVG seiner

12 Persson (1992: 158) bestreitet gegenüber von Polenz (1987) jedoch, dass Kausative wie zum Lachen, zum Kochen bringen usw. überhaupt als FVG anzusehen sind. 
Auffassung nach zwingend erforderlichen semantischen Mehrwert auf. Die entsprechenden Fügungen können daher in der Regel durch ein bedeutungsgleiches einfaches Verb ersetzt werden, vgl. (6) und (7).

(6) a. Er hat sein Bedauern ausgedrückt.

b. Er hat sein Bedauern zum Ausdruck gebracht.

(7) a. Er hat das Stück aufgeführt.

b. Er hat das Stück zur Aufführung gebracht.

Da bringen in Fällen wie diesen „keine spezifische eigene semantische Funktion“ (von Polenz 1987: 184) ausübe, handele es sich nicht um ein FV und bei der betreffende Konstruktion folglich nicht um ein FVG. Vielmehr diene bringen hier ,in erster Linie der nominalisierenden (substantivierenden) Ausdrucksweise, die sprachkritisch negativ beurteilt wird als ,Papierdeutsch', ,Substantivitis“, ,Verbaufspaltung““ (ebd.). Statt als FV sieht von Polenz bringen in diesem und vergleichbaren Fällen schlicht als „Nominalisierungsverb“ an. Dementsprechend werden bei von Polenz solche FVG, die einen kausativen oder einen inchoativen Bedeutungsaspekt gegenüber dem einfachen Verb ausdrücken, von sog. „Nominalisierungsverbgefügen“ abgegrenzt (vgl. dazu aus sprachhistorischer Perspektive auch von Polenz 1999: 352). Im Gegensatz von „Nominalisierungsverbgefüge“ (NVG) und FVG scheint somit letztlich immer noch die sprachkritisch motivierte Unterscheidung zwischen den notwendigen und (angeblich) überflüssigen Nomen-Verb-Verbindungen durch.

Im Abgrenzung zu von Polenz (1987) plädiert Seifert (2004: 75-78) ausdrücklich dafür, auch „Pseudokausativa“ als FVG zu behandeln, da diese gegenüber dem einfachen Verb häufig eben doch einen semantischen Mehrwert besäßen. So akzentuiere ein Pseudokausativum wie zum Abschluss bringen gegenüber einfachem abschließen die „Vorphase“ einer Handlung, beinhalte somit einen Wechsel der Aktionsart. ${ }^{13}$ Zudem könne durch Verwendung des Kausativums zum Abschluss bringen eine gewisse „Nachhaltigkeit“ betont werden, mit der eine Handlung durchgeführt werde, vgl. eine Dissertation zum Abschluss bringen, das gegenüber einfachem eine Dissertation abschließen gewisse Schwierigkeiten und Mühen bei der Ausführung der Handlung andeute. Seifert setzt diese „Nachhaltigkeit“ des FVG mit inchoativer Aktionsart gleich, da hier „die Vorphase der Handlung in den Blickpunkt tritt“ (2004: 77). Genau diese Gleichsetzung ist indes problematisch: Inchoativität beinhaltet den Übergang in einen anderen Zustand

13 In diesem Sinn vgl. aber auch schon von Polenz (1963: 42). 
des Aktanten (vgl. grundlegend Steinitz 1981: 9); ein Übergang in einen neuen Zustand ,abgeschlossen sein“ gilt aber sowohl für das einfache Verb abschließen wie für die Fügung zum Abschluss bringen. Eine Aktionsartenopposition zwischen Verb und FVG liegt hier also gerade nicht vor. Trotzdem ist Seifert insofern zuzustimmen, als zum Abschluss bringen in der Tat tendenziell eine größeres Zeitintervall zwischen Beginn und Ende der Handlung zu implizieren scheint und einfaches abschließen demgegenüber eher einen punktuellen Umschlag von einem Vorzustand in einen Nachzustand ausdrückt. Auf diese Differenzierung wird in Kap. 3 noch einzugehen sein.

Anderen Pseudokausativen, z. B. zum Ausdruck, in Vorschlag bringen, spricht Seifert (2004: 78f.) jedoch keinen semantischen Mehrwert gegenüber den entsprechenden einfachen Verben zu, da dort keine Betonung der Vorphase erkennbar sei. Aufgrund der fehlenden semantischen Eigenleistung wird solchen Formen - noch ganz im Sinne der normativen Sprachkritik der 1960er Jahre lediglich der Status einer „Streckform“ zuerkannt. Die „Streckformen“ entsprächen dann der Klasse der Nominalisierungsverbgefüge, die von Polenz (1987) für solche angeblich funktionslosen Fügungen eingeführt hatte. Aus der Klasse der FVG will Seifert solche „Streckformen“ gleichwohl nicht ausschließen. Unklar bleibt dabei aber letztlich, welche anderen Gesichtspunkte die Aufnahme in die Klasse der FVG rechtfertigen, da doch auch für Seifert (2004) der semantische Mehrwert das entscheidende Kriterium zu sein scheint.

Für die kausative Funktion ist somit ein ähnliches Fazit zu ziehen wie für die angebliche inchoative Funktion. Viele Bildungen, d. h. insbesondere die ,pseudokausativen“ Bildungen mit bringen in (4), haben keinen klar erkennbaren Mehrwert gegenüber den entsprechenden einfachen Verben. Man muss sie entweder aus der Klasse der FVG ausschließen, wie von Polenz es konsequenterweise tut, oder sie trotzdem als FVG behandeln und dementsprechend nach anderen Kriterien suchen, die eine solche Zuordnung rechtfertigen. Vorschläge, die in die letztgenannte Richtung gehen, werden weiter unten in Abschnitt 2.2.5 behandelt.

\subsubsection{Passivische FVG}

Im Zentrum der Klasse FVG stehen traditionellerweise die als inchoativ bzw. kausativ deklarierten Fügungen. Zu den FVG werden seit von Polenz (1963: 20), Daniels (1963: 52) und Kolb (1965/1966) häufig aber auch Verbindungen wie Anerkennung, Berücksichtigung, Verwendung finden oder Vereinfachung, Veränderung, 
Erweiterung erfahren gerechnet, denen jeweils Passivformen mit dem Hilfsverb werden entsprechen: ${ }^{14}$

(8) a. Ihre Arbeit findet Anerkennung.

b. Ihre Arbeit wird anerkannt.

(9) a. Dieser Punkt findet keine Berücksichtigung.

b. Dieser Punkt wird nicht berücksichtigt.

(10) a. Schon wenig später erfuhr die Wallfahrtskapelle eine wichtige Erweiterung: Hermann von Stein [...] stiftete ein Leprosenhaus. Nürnberger Nachrichten, 05.10.2012, S. 10; DEREKo, Zugriff 07.11.2014) ${ }^{15}$

b. Schon wenig später wurde die Wallfahrtskapelle erweitert.

von Polenz (1987: 170) sieht hierin ,einen speziellen Typ von FVG, in denen weder Kausativität noch eine Aktionsart im Spiel ist, sondern nur Passivität, so dass diese FV den gleichen semantischen Status haben wie die PASSIV-Hilfsverben werden und bekommen“. Da die passivischen FVG stets durch ein werden-Passiv ersetzbar seien, erkennt von Polenz diesen Fügungen offenbar keinen semantischen Mehrwert gegenüber den etablierten Ausdrucksmitteln für das Passiv zu. Dies mag der Grund dafür sein, dass von Polenz in späteren Arbeiten die betreffenden Verbindungen nicht mehr als FVG klassifiziert, sondern lediglich ,in die Nähe der FVG“ (1999: 352) rückt (vgl. auch 1988: 185).

Andere Forscher gehen indes sehr wohl von einem systematischen semantischen Mehrwert von Fügungen mit finden/erfahren aus und schlagen die genannten Fügungen den FVG zu. ${ }^{16}$ So hat Daniels (1963: 20) in einer „Erfolgsbetonung“, d. h. in einer ausgeprägten resultativen Bedeutungskomponente, eine besondere

14 Auch bei Pape-Müller (1980: 23), Helbig (1984: 177), Zifonun/Hoffmann/Strecker (1997: 53), Eroms (2000: 168f.) werden Konstruktionen mit finden/erfahren als passivische FVG behandelt. - Rösch (1994) hat neben dem Kernbereich der passivischen FVG, die eine äquivalente werdenKonstruktion neben sich haben, noch eine weitere Gruppe sog. ,passivwertiger“ FVG angesetzt, denen kein werden-Passiv zur Seite steht (z. B. Niederschlag, Beifall finden). Nach welchen Kriterien diese Verbindungen als FVG behandelt werden, lässt Rösch jedoch im Unklaren, vgl. auch die Kritik van Pottelberges (2001: 442-444). Daher sei hier nicht weiter darauf eingegangen.

15 Im Folgenden werden die Belegzitate, die aus dem DeReKo (dem deutschen Referenzkorpus des IDS Mannheim) entnommen sind, nicht mehr eigens gekennzeichnet.

16 Eine besondere Leistung der Fügungen mit finden und erfahren haben Daniels (1963: 212) und Guttmacher (1980: 107) in der Möglichkeit gesehen, zu intransitiven Verben ein persönliches Passiv zu bilden, z. B. unterschlüpfen - Unterschlupf finden, gefallen - Gefallen finden, zusammenbrechen - einen Zusammenbruch erfahren/erleiden. Aufgrund der begrenzten Zahl solcher Bildungen ist jedoch von keiner systematischen Leistung auszugehen. 
Eigenschaft der Verbindungen mit finden gesehen, und auch Guttmacher (1980: 107f.) spricht ganz in diesem Sinne von einer „Zustandsveränderung mit Ergebnisbetonung“, die durch das „resultatsbezogene perfektive FV ,finden““ ausgedrückt werde. Einen spezifischen aktionalen Gehalt der genannten FVG erkennt auch Persson (1981: 27), nach dessen Auffassung die Aufgabe des FV finden darin bestehe, „die transformative Aktionsart des Prädikats [...] zu stärken“. Allen genannten Autoren fällt es aber offensichtlich schwer, einen wirklich greifbaren Unterschied zwischen werden-Passiva und korrespondierenden FVG herauszuarbeiten; sie sprechen daher auffälligerweise stets von der ,Betonung bzw. ,Stärkung' der resultativen Komponente, die bei werden-Passiv somit zwar vorhanden, aber nur nicht so deutlich erkennbar sei.

Ein Grund dafür, dass vielfach nach einem Unterschied zwischen einem finden-FVG und einem entsprechenden Passiv gesucht wird, mag darin zu sehen sein, dass finden weniger stark desemantisiert ist als das Hilfsverb werden. Insgesamt ist ein klar erkennbarer semantischer Mehrwert der finden-FVG gegenüber den parallelen werden-Konstruktionen jedenfalls nicht erkennbar. Solange man einen systematisch beschreibbaren Mehrwert der Nomen-Verb-Verbindung gegenüber einem existierenden Ausdrucksmittel als Definitionskriterium für FVG ansieht, ist von Polenz (1999: 352) somit zuzustimmen, wenn er die finden-Fügungen nicht als FVG auffasst.

\subsubsection{Systematizität der FVG}

\subsubsection{1 Übereinstimmung von Form und Funktion}

Während die grammatische Funktion der Aktionsarten- bzw. Passivmarkierung in der älteren Forschung - etwa bei Kolb (1962), von Polenz (1963) und Daniels (1963) - noch das zentrale Kriterium für den Ansatz der Klasse FVG war, haben vor allem die Arbeiten von Helbig (1984) und von Polenz (1987) einen anderen Aspekt in der Vordergrund gerückt (dies wohl durchaus in dem Bewusstsein, dass die bisherigen Definitionsversuche ihre Grenzen haben), nämlich die „systematisch beschreibbare Eigenbedeutung in Gruppen“ von Nomen-Verb-Verbindung (von Polenz 1987: 170; vgl. auch Helbig 1984: 167f., 179). ${ }^{17}$ von Polenz und Helbig zielen damit auf eine Eins-zu-Eins-Zuordnung von Form und Funktion als besonderer Eigenschaft der FV ab, wie sie auch bei den Hilfs- und Modalverben

17 In eine ähnliche Richtung gehen bereits die Untersuchungen von Heringer (1968), Engelen (1968), Klein (1968), Herrlitz (1973) und Persson (1975). 
zu finden ist: Die Verben bringen, kommen oder setzen würden systematisch dafür genutzt, jeweils eigene grammatische Funktionen auszudrücken (zur Analogie mit den Hilfs- und Modalverben von Polenz 1987: 170; Eroms 2000: 165) ${ }^{18}$ In dieser konsequenten Eins-zu-eins-Zuordnung von Form und Funktion, die FVG im Gegensatz zu einfachen Verben ermöglichen, wird ein Mehrwert gesehen, der FVG eine Existenzberechtigung verschafft.

Indem hier das Kriterium der Systematik des durch das Verb geleisteten Bedeutungsbeitrags in den Mittelpunkt gerückt wird, ist das Problem allerdings nur bedingt entschärft. Zwar ist teilweise in der Tat ein relativ hohes Maß an Systematik in der Markierung bestimmter Funktionen erkennbar, wie z. B. die Kennzeichnung des Inchoativums durch kommen oder geraten zeigt (zu den Beispielen s. auch Helbig 1984: 179 und von Polenz 1987: 172-175):

(11) a. in Bewegung kommen, in Gang kommen, in Umlauf kommen, ins Grübeln kommen, zur Darstellung kommen

b. in Aufregung geraten, in Vergessenheit geraten, in Brand geraten, ins Rutschen geraten

Wenn man, wie vor allem von Polenz (1987) propagiert hat, die Regelhaftigkeit in der Entsprechung von Form und Funktion zum Kriterium für den Ansatz einer Klasse FVG erhebt, zieht dies allerdings die Frage nach sich, wie oft ein Verb für eine bestimmte Funktion genutzt werden muss, damit von Systematizität die Rede sein kann. von Polenz (1987: 176) will vereinzelt in Kombination mit einem Nomen auftretende Verben (er nennt $\mathrm{u}$. a. ins Leben rufen oder an Bedeutung gewinnen) aus dem Bestand der FVG ausschließen. Verbindungen mit gehen, für die es relativ wenige Beispiele gibt, werden bei von Polenz gleichwohl als FVG klassifiziert:

in Arbeit gehen, in Druck gehen, in Revision gehen

(vgl. von Polenz 1987: 174)

Es bleibt somit unklar, wo hier die Grenze zu ziehen ist, damit von einem ,systematischen Bedeutungsbeitrag“ des Verbs die Rede sein kann. Wenn von Polenz (1987: 176) in Anbetracht dieser Schwierigkeit „einen breiten Übergangsbereich“ zwischen FVG und idiomatischen Nomen-Verb-Verbindungen postuliert, hat dies den Charakter einer Verlegenheitslösung.

18 In die Nähe der Hilfsverben werden die FV bereits bei Kolb (1962: 381) und von Polenz (1963: 13f., 16f.) gerückt, vgl. ferner Herrlitz (1973: 18), Helbig (1983: 175), Flämig (1991: 179). 
Vorbehalte gegenüber der behaupteten Analogie der FVG zu den Hilfs- und Modalverben ergeben sich aber noch von anderer Seite her: Die Kodierung des Passivs durch werden oder der deontischen Modalität durch sollen ist wesentlich fester im Sprachsystem verankert, als die Kodierung der inchoativen Aktionsart durch kommen oder gelangen. Hier gibt es, wie die Beispiele in (13) zeigen, zahlreiche Ausdrucksalternativen, wie sie für ,echte‘ Hilfs- bzw. Modalverben in diesem Ausmaß nicht zur Verfügung stehen. Hier kann folglich von der exklusiven Verknüpfung einer Form mit einer Funktion nicht die Rede sein.
a. aufnehmen (Arbeit, den Kampf, Verhandlungen)
b. fallen (in Ohnmacht, Ungnade, Ungunst)
c. gehen (in Druck, Arbeit)
d. treten (ins Bewusstsein, in Kraft)
e. nehmen (in Gebrauch, Besitz, Verwahrung)
f. ausbrechen (in Jubel)
g. schreiten (zur Arbeit, Tat)

Hilfsverben und auch Modalverben zeichnen sich jedenfalls durch weniger Ausdrucksalternativen aus, so dass der häufig gezogene Vergleich zwischen diesen und den FV kaum weiterhilft.

\subsubsection{Reihenbildung}

Ein wichtiges Kriterium für den Ansatz von FVG stellt auch die systematische Bildung von Oppositionen, die sog. „Reihenbildung“ (von Polenz 1987: 175; Eisenberg 2006a: 314), dar (vgl. auch Engelen 1968: 293; Helbig 1984: 169; Seifert 2004: 57). Solche regelhaft auftretenden Relationen bestehen etwa zwischen kausativem bringen oder setzen/versetzen, inchoativem kommen oder geraten und durativem sein bzw. sich befinden, vgl. die Fügungen in (14):

(14) a. in Abhängigkeit bringen - kommen/geraten - sich in Abhängigkeit befinden

b. in Bewegung setzen - geraten/kommen - sein

c. in Aufregung versetzen - geraten - sein 
Mit dem Merkmal der Reihenbildung werden FV deutlich in die Nähe der Hilfsverben gerückt. Diese zeichnen sich ebenfalls dadurch aus, dass sie in geschlossenen Subklassen auftreten (z. B. als Hilfsverben der Tempusbildung oder der Diathese), die sich durch systematische funktionale Oppositionen auszeichnen.

Die Reihenbildung erweist sich bei näherer Betrachtung freilich als ein wenig brauchbares Abgrenzungskriterium. So ist eine mit den Fällen in (14) vergleichbare Reihenbildung bei den Kausativa mit bringen + Infinitiv, die von Polenz (1987: 182f.) explizit zu den FVG rechnet, gerade nicht gegeben, vgl. (15).

(15) a. zum Schweigen bringen - ?zum Schweigen kommen - *im Schweigen sein

b. zum Lachen bringen - ?zum Lachen kommen - *im Lachen sein

c. zum Kochen bringen - ?zum Kochen kommen - *im Kochen sein

Die kommen-Verbindungen in (15) sind zwar teilweise durchaus bildbar, jedoch nicht als Inchoativa, sondern in einer anderen Bedeutung, nämlich ,Zeit, Gelegenheit haben (etwas zu tun)` wie in (16).

(16) Er erzählte einen Witz nach dem anderen, da sind wir gar nicht zum Lachen gekommen.

Irgendeine signifikante semantische Relation zwischen kausativem zum Lachen bringen und der kommen-Verbindung in (16) besteht nicht.

Reihenbildung scheint also, wie die hier behandelten Beispiele zeigen, kein notwendiges Kriterium für eine Klasse ,FVG` zu sein. Dass es sich ebenso wenig um ein hinreichendes Kriterium handelt, hat van Pottelberge (2001: 231) an der Reihenbildung gezeigt, die man auch für die „Vollverben“19 bringen, kommen und sein postulieren kann; vgl. dazu das folgende Beispiel:

(17) ins Krankenhaus bringen - ins Krankenhaus kommen - im Krankenhaus

19 Mit dem Begriff „Vollverb“ werden alle Verben bezeichnet, die nicht Hilfs-, Modal oder eben Funktionsverben sind (vgl. Zifonun/Hofmann/Strecker 1997: 50-52). 
Ungeeignet für eine Klassenabgrenzung ist das Kriterium der Reihenbildung auch deshalb, weil solche Reihen in großer Zahl auch bei Phraseologismen auftreten, vgl. hier die Beispiele in (18). ${ }^{20}$
a. in Harnisch bringen - geraten - sein
b. aus dem Häuschen bringen - geraten - sein
c. hinter Gitter bringen - kommen - hinter Gittern sein
d. unter die Haube bringen - kommen - unter der Haube sein
e. ins Lot bringen - kommen - im Lot sein

Nun könnte man freilich auch Phraseologismen wie die in (18) aufgrund der hier vorliegenden Reihenbildung als FVG behandeln. In diesem Fall ergibt sich jedoch die Schwierigkeit, dass den solchermaßen als FVG deklarierten Phraseologismen eine zentrale Eigenschaft aller übrigen FVG, nämlich das Vorhandensein eines Verbalnomens, fehlte. Wohl aus diesem Grund hat die FVG-Forschung diesen Weg bisher kaum oder zumindest nicht konsequent beschritten. ${ }^{21}$

\subsubsection{Weitere Funktionen}

Der sprachliche Mehrwert von FVG wird nicht nur in der Möglichkeit einer systematischen Markierung grammatischer Funktionen gesehen, sondern auch in zahlreichen weiteren Leistungen, die eher stilistischer Natur sind (vgl. die Zusammenstellung stilistischer Funktionen bei Helbig 1984: 176f., Yuan 1987: 196207 und Seifert 2004: 105-107). Dazu gehören z. B. die Möglichkeit der Tilgung eines Aktanten bei transitiven Vollverben, die angeblich eine ,allgemeinere Bedeutung“ (Helbig 1984: 177) haben, s. (19).

\footnotetext{
20 Auch Burger/Buhofer/Sialm (1982: 37) und van Pottelberge (2001: 233) betonen die Verwandtschaft solcher Phraseologismen mit den FVG, vgl. auch Rostila (2011: 272).

21 Lediglich vereinzelt werden Phraseologismen den FVG zugeschlagen, ohne dass dies jedoch angemessen begründet oder konsequent durchgeführt wird, vgl. Engelen (1968: 295); Klein (1968: 9); Rothkegel (1973: 54); Herrlitz (1973: 16f., 161f.); Rostila (2011: 272); mit Begründung Kamber (2008: 31f.). von Polenz (1987: 175) hat einzelne Phraseologismen (auf die Palme bringen) als FVG interpretiert, da die Nominalgruppe semantisch als nomen actionis zu deuten sei: auf die Palme entspreche ,in Wut' (s. o.). Im Gegensatz dazu wird bei von Polenz (1988: 884) auf die Palme bringen allerdings lediglich dem Übergangsbereich zwischen idiomatischen Verbindungen und FVG zugerechnet. $\mathrm{Zu}$ diesem Problem vgl. auch Kap. 3.2.3.
} 
Wir stiften Verwirrung vs. 'Wir verwirren.

Auch die Möglichkeit der Attribution des Nomens, die einige FVG bieten, kann nach Helbig (1984: 177) und Seifert (2004: 105f.) ein Vorteil gegenüber der Versprachlichung durch ein einfaches Verb sein, da letztere häufig keine oder nur eine relativ umständliche Modifikation des Ereignisses erlaubt. Dies zeigt z. B. das etwas ungeschickte Adverbial in (20a) oder der Nebensatz in (20b) gegenüber der unkomplizierten Modifikation in (20c), die ein Satz mit FVG ermöglicht.

(20) a. Nach langen Jahren wurde die Leistung verdientermaßen anerkannt.

b. Nach langen Jahren wurde die Leistung des Künstlers endlich anerkannt, wie sie es verdient hat.

c. Nach langen Jahren fand die Leistung des Künstlers endlich die verdiente Anerkennung.

Eine häufig genannte kommunikative Leistung der FVG besteht in der Modifikation der Thema-Rhema-Struktur eines Textes durch die Satzklammer (vgl. Helbig 1984: 177; von Polenz 1987: 170; Seifert 2004: 106). Die Aufspaltung des Prädikatsgefüges in einen verbalen und einen nominalen Teil eröffnet die Möglichkeit, dass „der sinnwichtigste Teil als Rhema (Neumitzuteilendes) wirkungsvoll hochtonig am Satzende steht“ (von Polenz 1987: 170), vgl. (21).

(21) a. Die Landwirtschaft der DDR entwickelt sich gut.

b. Die Landwirtschaft der DDR nimmt eine gute Entwicklung. ${ }^{22}$

(Helbig 1984: 177)

Im Gegensatz vor allem zur Aktionsartenmarkierung werden die hier beispielhaft aufgeführten stilistischen Funktionen jedoch eher selten als klassenkonstituierende Merkmale angesehen (vgl. von Polenz 1987: 170). Lediglich Seifert (2004: 92f.) scheint - neben dem angeblichen grammatischen Mehrwert der Kodierung von Aktionsarten, Kausativ und Passiv - konsequent auch das Vorhandensein eines stilistischen Mehrwerts als Definitionskriterium für FVG anzusetzen. Dies

22 An diesem Beispiel zeigt sich der postulierte Einfluss des FVG auf die Thema-Rhema-Struktur jedoch gerade nicht, da in beiden Sätzen das Rhema am Ende des Satzes steht. Dieser Einwand gilt nicht nur für das oben genannte Satzpaar, sondern scheint ein grundsätzliches Problem dieser Hypothese zu sein, vgl. die Sätze Er entscheidet vs. Er bringt zur Entscheidung, die ebenfalls identische Thema-Rhema-Verteilung aufweisen. 
führt jedoch, wie Seifert selbst anmerkt, zu schwierigen Einzelfallentscheidungen. Im Ganzen wird man daher den stilistischen Funktionen keine tragende Rolle bei der Frage nach der Klassenabgrenzung zumessen wollen (aus anderer Perspektive dazu Kap. 7).

\subsection{Die syntaktische Struktur von FVG}

\subsubsection{Die verbale und nominale Komponente}

Wenn auch FVG insgesamt eine sehr umstrittene Klasse darstellen, so herrscht doch über eine Eigenschaft von FVG Konsens in der Forschung: Das Funktionsverb ist im Vergleich zum „Vollverb“ durch den Verlust seiner eigentlichen, konkreten Bedeutung gekennzeichnet. ${ }^{23}$ Es wird daher im allgemeinen als „,bedeutungsarm“, „inhaltsarm“, „abstrakt“ charakterisiert (Daniels 1963: 21; vgl. von Polenz 1963: 26; 1987: 169) ${ }^{24}$ oder als „weitgehend einer selbständigen Bedeutung entleert“ (Busse 2002: 412), „verblaßt“ (Zifonun/Hoffmann/Strecker 1997: 53), „bedeutungsschwach“ (van Pottelberge 2001: 55), als „bis zu einem gewissen Grade desemantisiert“ (Seifert 2004: 55) beschrieben. Als verbindendes Merkmal aller Beschreibungen der Semantik von FV gilt aber auch, dass nicht versucht wird, eine Explizierung der weitgehend intuitiv verwendeten Begriffe „inhaltsarm“, „verblasst“, „,ausgeblichen“ usw. zu leisten. Dabei bleiben indes zentrale Fragen unbeantwortet: Wie weit muss diese „Inhaltsarmut“ gehen, damit von einem FV die Rede sein kann? Wie kann man die „Inhaltsarmut“ methodisch einigermaßen sicher bestimmen?

Während in der Charakterisierung des Verbs als „inhaltsarm“ o. ä. weitgehend Einigkeit herrscht, ist der nominale Bestandteil wesentlich umstrittener. ${ }^{25}$

23 Dass das FV im Gegensatz zum FVG als Ganzem relativ problemlos zu fassen sei, bestätigt auch Eisenberg (2006a: 309): „Der Status dieses Begriffs [FVG, V. H.] ist viel unklarer als der des Funktionsverbs selbst“.

24 Daniels (1963: 21) weist darauf hin, dass die semantische Reduktion des Verbs in nominalen Fügungen schon in älterer Literatur bemerkt worden ist, vgl. Wunderlich (1901: 24), Schneider (1925: 778).

25 Detges (1996: 4) setzt FVG mit „komplexen Prädikatsausdrücken“ gleich. Als „komplexe Prädikatsausdrücke“ sind auch Verbindungen eines Adjektivs mit einem Verb zu behandeln (krank machen, bekannt geben, fündig werden). Im Allgemeinen werden jedoch Adjektiv-Verbindungen nicht als FVG behandelt (vgl. lediglich Cattell 1984: 175 für das Englische). van Pottelberge (2001: 15f.) macht zu Recht darauf aufmerksam, dass diese Trennung weniger sachliche Gründe hat, sondern vielmehr hauptsächlich einer einmal festgelegten Forschungstradition folgt. 
Zwar gibt es auch hier insofern einen kleinsten gemeinsamen Nenner, als Ausdrücke für Konkreta (Haus, Stein, Pferd) generell nicht als Nomina in FVG behandelt werden. Dieser gemeinsame Nenner trägt jedoch nicht weit, da die Auffassungen darüber, was denn sonst als nominaler Bestandteil vorkommen kann, beträchtlich auseinandergehen. Von einem kleineren Teil der Forschung wird der nominale Teil auf Verbalabstrakta beschränkt (von Polenz 1963: 11; Burger/Buhofer/Sialm 1982: 37; Persson 1992: 156; Fleischer 1997: 137), ein größerer Teil der Forschung bezieht auch deadjektivische Ableitungen mit ein, da auch hier Reihenbildung und systematischer Bedeutungsbeitrag des FV gegeben sind, z. B. in Abhängigkeit geraten, bringen, sein (vgl. Helbig 1984: 165; von Polenz 1987: 171, vgl. auch Heringer 1968: 23-32; Herrlitz 1973: 16f.; Seifert 2004: 57).

Um eine präzise Bestimmung des nominalen Elements in FVG hat sich vor allem Heringer (1968) verdient gemacht. ${ }^{26}$ Heringer bezeichnet die nominale Komponente in FVG im Anschluss an von Polenz (1963: 11) als nomen actionis. Dieses wird wie folgt definiert:

Ein Nomen actionis besitzt die gleiche semantische Funktion wie ein Verbum: es bezeichnet eine Handlung, einen Vorgang oder einen Zustand. Dies kommt allerdings in dem Zusatz ,actionis‘ nicht klar zum Ausdruck, da wir kein Wort besitzen, das diese drei Funktionen zusammen bezeichnet.

(Heringer 1968: 25f.)

Heringer (1968: 27) versteht nomen actionis jedoch ausdrücklich nicht morphologisch als Deverbativum, sondern fasst den Begriff rein inhaltsseitig: So sei Ruhe zwar kein Verbalabstraktum zu ruhen, dennoch bestehe ein synchroner semantischer Zusammenhang mit dem Verb ruhen, das historisch gesehen von Ruhe abgeleitet sei. Dieser Zusammenhang „genügt für jeden Sprachteilhaber, in der Wendung zur Ruhe kommen das Wort Ruhe als Nomen actionis zu empfinden“ (1968: 27). Im Weiteren werden nicht nur Infinitive, suffixlose und isolierte Verbalabstrakta (Lehnwörter eingeschlossen) sowie -ung-Ableitungen, sondern auch Bildungen auf -nis-, -heit, -keit, -schaft ausdrücklich zu den formalen Realisationsmöglichkeiten eines nomen actionis gerechnet, obwohl die zuletzt genannte Gruppe durchaus nicht nur zu Verben gebildet wird (1968: 27f.). (In den konkreten Beispielen, die Heringer in seiner Untersuchung bietet, finden sich dann aber keine deadjektivischen oder desubstantivischen Bildungen als nomina actionis in FVG.) Konkreta, wie sie in zu Papier bringen, zu Geld kommen, in Verse

26 Noch bei Seifert (2004: 56) wird Heringers Begriffsbestimmung als „tragfähig“ angesehen und übernommen. 
bringen vorliegen, schließt Heringer allerdings aus, obwohl auch sie gemeinsam mit dem Verb eine den FVG vergleichbare Einheit bilden. ${ }^{27}$

Die von Heringer vollzogene Abkehr von einer morphologischen Bestimmung und die Hinwendung zu einer rein inhaltsseitigen Definition des Nomens in FVG ist von der Forschung weitgehend übernommen worden. Vor allem von Polenz (1987) hat den inhaltsseitigen Ansatz Heringers konsequent weiterzuführen versucht. So klassifiziert von Polenz $(1987: 175,183)$ auch die Substantive in aus dem Konzept, auf die Palme, zur Weißglut bringen, in Harnisch geraten als nomina actionis, obwohl keine synchrone Ableitungsrelation zu einem Verb oder Adjektiv vorliegt. Begründet wird dies damit, dass mit diesen Substantiven eine Handlung, eine Tätigkeit, ein Ereignis, ein Vorgang oder ein Zustand bezeichnet werde. ${ }^{28}$ So sei auf die Palme als ein Zustand ,in Wut' und daher als nomen actionis zu interpretieren. Hier stellt sich allerdings die Frage, ob es berechtigt ist, die Substantive in etwas zu Papier, an den Tag, an die Öffentlichkeit, auf die Bühne, etwas nicht über die Lippen bringen ausdrücklich nicht als nomina actionis zu klassifizieren (1987: 175, 181). Auch die Bedeutung von zu Papier kann als ,in schriftliche Form', d. h. als Zustand, gedeutet werden; ebenso kann auf die Bühne als Zustandsbeschreibung ,aufgeführt', über die Lippen als Zustand ,ausgesprochen' usw. gelesen werden. Wenn man sich, wie Heringer und von Polenz, einmal auf die rein inhaltsseitige Bestimmung des nomen actionis ohne Rücksicht auf das Bestehen synchroner Ableitungsverhältnisse einlässt, fällt es somit schwer, Substantive in Verbverbindungen zu benennen, die sich eindeutig nicht als Zustandsbezeichnungen interpretieren lassen. ${ }^{29} \mathrm{Geht}$ man den von Heringer eingeschlagenen Weg konsequent zu Ende, wären in der Tat auch die genannten

27 Eine Schwierigkeit ergibt sich jedoch dadurch, dass Heringer (1968: 27f.) ausdrücklich auch die isolierten, d. h. synchron nicht motivierbaren Ableitungen zur Gruppe möglicher nomina actionis rechnet (Beispiel in Angriff nehmen). Einige dieser isolierten Ableitungen wie Angriff, die mit angreifen und der homonymen Ableitung Angriff nicht mehr in Verbindung stehen, sind indes oftmals nur schwer als Handlungs-, Vorgangs- oder Zustandsbezeichnungen beschreibbar, da ihnen mangels semantischer Motivation keine eigene Bedeutung zugesprochen werden kann. Ähnlich schwierig ist Sprache in der Verbindung zur Sprache kommen/bringen - nach Heringer (1968: 26) ein FVG. Ob Sprache hier einen Vorgang, Zustand oder eine Handlung bezeichnet und somit als nomen actionis im Sinne Heringers aufzufassen ist, darf bezweifelt werden.

28 von Polenz (1987: 175) spricht etwas missverständlich davon, dass auf die Palme, zur Weißglut usw. „referenzlos“ seien. Natürlich haben auch solche Substantivgruppen keine Referenz insofern, als sie sich auf einen Sachverhalt in der Welt beziehen. Mit „referenzlos“ ist wohl lediglich ,nicht-konkret' gemeint, vgl. auch van Pottelberge (2001: 234).

29 Die Schwierigkeit, dieser Begriffsbestimmung folgend Phraseologismen und FVG voneinander abzugrenzen, zeigt sich auch darin, dass von Polenz (1987) bringen in auf die Bühne bringen mal als idiomatisiertes Vollverb (181), mal als FVG ansieht (183). 
Phraseologismen als FVG zu behandeln. Die Grenze zwischen FVG und Phraseologismen würde sich damit aber endgültig auflösen. Diese Konsequenz hat die Forschung, die von Heringers Auffassung ausgeht, bisher offenbar nicht bedacht.

Statt einer inhaltlichen Bestimmung der nominalen Komponente von FVG ist jedoch auch eine strikt morphologische Herangehensweise propagiert worden. So besteht vor allem Fleischer (1997: 137) auf dem Vorhandensein eines ,echten“ nomen actionis, d. h. eines synchron auf eine verbale Basis beziehbaren Substantivs (vgl. auch Persson 1992: 157, van Pottelberge 2001: 234): ${ }^{30}$

Da als eine Bedingung für die Qualifizierung als Funktionsverbgefüge gilt, daß das Nomen ein Nomen actionis ist, erscheint es nicht gerechtfertigt, in Fällen, wo kein entsprechendes Verb mehr vorhanden ist oder das Substantiv überhaupt nur in der betreffenden Konstruktion verwendet wird, von einem Funktionsverbgefüge zu sprechen. Das gilt auch für die Fälle, wo die semantische Beziehung des Verbalsubstantivs zu dem zugrunde liegenden Verb verloren gegangen ist.

(Fleischer 1997: 137)

Verbindungen wie in Misskredit bringen, in Angriff nehmen, zum Vorschein bringen werden von Fleischer daher nicht als FVG, sondern als „Phraseolexeme“ bezeichnet.

Eine morphologische Definition des nominalen Bestandteils von FVG vertritt auch Helbig (1984: 168f.). Im Gegensatz zu der Auffassung etwa bei Fleischer (1997: 137) kann es sich Helbig zufolge bei den Substantiven in FVG jedoch nicht nur um deverbale, sondern auch um deadjektivische Ableitungen handeln (z. B. in Verlegenheit bringen). In der Umsetzung dieses Postulats zeigt sich Helbig allerdings wenig konsequent. So werden z. B. Mut, Angst haben sowie zu Ende bringen als FVG behandelt (1984: 169, 179), obwohl weder Mut, Angst noch Ende Ableitungen von Adjektiven bzw. Verben darstellen. Bei der Bestimmung von FVG und ihren Substantiven scheint sich Helbig in der Praxis somit eher an ein inhaltliches Bestimmungskriterium zu halten. ${ }^{31}$ Damit entspricht seine Herangehensweise weitgehend der eben geschilderten Sicht von Heringer (1968) und von Polenz (1987).

30 Seifert (2004) bezieht keine klare Position: Zwar wird grundsätzlich Heringers Auffassung zugestimmt (56); an anderer Stelle (61-64) schließt sich der Autor jedoch ausdrücklich der Position Fleischers an.

31 In ähnlicher Weise ambivalent zeigt sich auch Engelen (1968: 292), der zwar ausdrücklich festlegt: „Das Nomen des Funktionsverbgefüges ist ein Verbalabstraktum“, in seinen Beispielen (z. B. 1968: 295) jedoch auch Nomina nennt, die eindeutig keine Verbalabstrakta darstellen (Wut, Zorn). 
Welche der beiden geschilderten Herangehensweisen an das Substantiv von FVG - die semantische oder die morphologische - grundsätzlich als angemessen $\mathrm{zu}$ bewerten ist, hängt von übergeordneten Gesichtspunkten ab. Bevor dessen nominale Komponente näher charakterisiert werden kann, müsste geklärt werden, was ein FVG als Ganzes ausmacht.

\subsubsection{Die Struktur der Nominalgruppe}

Während über die beschriebenen Leistungen der FVG, vor allem über die zentrale Rolle der Aktionsartenmarkierung, weitgehend Einigkeit innerhalb der Forschung herrscht, ist hinsichtlich der syntaktischen Struktur der FVG bislang kein Konsens erreicht. Ungeklärt ist zunächst die Frage, ob (und wenn ja, in welchem Umfang) neben den präpositionalen Fügungen (zur Aufführung kommen), die übereinstimmend als FVG betrachtet werden, auch die akkusativischen Verbindungen (Kenntnis geben, Hilfe leisten, eine Entscheidung treffen/fällen) zu den FVG zu zählen sind. von Polenz (1963) behandelt im wesentlichen nur die präpositionalen Fügungen als FVG, ebenso Engelen (1968), Heringer (1968), Eisenberg (2006a) sowie weitgehend auch von Polenz (1987). Nach anderen Autoren (z. B. Klein 1968; Bahr 1977; Guttmacher 1980; Helbig 1984; Seifert 2004; Storrer 2006) stellen auch Verbindungen mit einem Nomen im Akkusativ FVG dar.

Als Grund für den Ausschluss der akkusativischen Fügungen wird bei von Polenz (1963) das Fehlen eines semantischen Mehrwerts gegenüber dem parallelen einfachen Verb genannt: Im Fall von eine Entscheidung treffen/fällen ,ist der Vorgangsbegriff ,entscheiden' gegenüber der rein verbalen Stilvariante (ich entscheide über etwas) nicht differenziert“ (1963: 13). Dagegen stecke hinter einer präpositionalen Fügung ich bringe etwas zur Entscheidung „mehr als eine bloße Umschreibung des Vorgangsbegriffs“ (ebd.), nämlich eine deutliche aktionale Differenzierung. ${ }^{32}$

Bei von Polenz (1987) werden akkusativische Fügungen offenbar auch deshalb aus dem Bestand der FVG ausgeschlossen, weil das Kriterium der ,systematisch beschreibbaren Eigenbedeutung in ganzen Gruppen' (s.o. 2.4) bei vielen dieser Fügungen nicht erfüllt ist. Damit fallen vor allem isoliert auftretende Verben wie (Lob) zollen oder (Verdacht) hegen aus der Gruppe der FV heraus. Dabei

32 Pape-Müller (1980: 24) rechnet akkusativische Verbindungen zwar durchaus zu den FVG, gesteht aber kategorisch nur präpositionalen Verbindungen die Möglichkeit der Aktionsartenmarkierung zu. Weshalb akkusativische Verbindungen nicht der Aktionsartenmarkierung dienen, wird hier, ebenso wenig wie sonst in der Forschung, nicht erklärt. 
übersieht von Polenz aber, dass es durchaus auch Verben gibt, die reihenhaft in Verbindung mit einem Nomen im Akkusativ auftreten. Diese entsprechen dem Kriterium der systematisch beschreibbaren Bedeutung in Gruppen daher durchaus, vgl. die Fälle in (22).

(22) a. eine Äußerung, einen Ausspruch, eine Frage, einen Schrei, einen Vorschlag (...) tun

b. Andeutungen, Anfang, Angaben, Anzeige, Ausgaben, Bemerkungen, Einkäufe, Erfindungen (...) machen

Wie in Abschnitt 2.4.2 gezeigt, wird auch die Bildung von systematischen Oppositionen wie bei kommen - bringen - sein als Charakteristikum von FVG genannt, vgl. die Beispiele in Tab. 1. Oppositionsbildung - zumindest im Ansatz - zeigen aber auch die akkusativischen Fügungen (Beispiele nach Guttmacher 1980: 124):

Tab. 1: Oppositionsbildung durch FV

\begin{tabular}{lll}
\hline durativ & inchoativ & kausativ \\
\hline Kenntnis haben & Kenntnis nehmen & Kenntnis geben \\
Veranlassung haben & Veranlassung nehmen & Veranlassung geben \\
Einblick haben & Einblick nehmen & Einblick geben \\
Abstand haben/halten & Abstand nehmen & \\
Fühlung haben/haben & Fühlung nehmen & \\
\hline
\end{tabular}

Das angebliche Fehlen systematischer Oppositionen kann den Ausschluss der oben genannten Bildungen aus der Klasse der FVG somit nicht rechtfertigen.

Neben präpositionalen und akkusativischen Fügungen werden gelegentlich auch Verbindungen mit einem nomen actionis im Nominativ als FVG klassifiziert oder zumindest in deren Nähe gerückt (vgl. von Polenz 1964: 1; Popadič 1971: 87; Bahr 1977: 18; Helbig 1984: 167f.; Helbig/Buscha 2001: 84; Seifert 2004: 69f.). Zu den wichtigsten Verben, die solche nominativischen FVG bilden, zählen nach Seifert (2004: 70) erfolgen, geschehen, stattfinden, nach Helbig (1984: 167f.) herrschen und bestehen: 
(23) a. Die Zahlung erfolgt per Kreditkarte.

b. Die Durchsicht erfolgt/geschieht gewissenhaft.

c. Die Untersuchung findet sofort statt/erfolgt sofort.

d. In dieser Frage herrscht/besteht Übereinstimmung.

Helbig (1984: 168f.) sieht eine Ähnlichkeit dieser Konstruktionen mit den FVG in der ansatzweise gegebenen Möglichkeit einer Oppositionsbildung wie z. B. in (24):

(24) a. Es herrscht Uneinigkeit. ${ }^{33}$ [= durativ]

b. Uneinigkeit entsteht/tritt ein/bricht aus/kommt zustande. [= inchoativ]

c. X schafft/macht/ruft Uneinigkeit hervor. [= kausativ]

Freilich stehe, so Helbig weiter, das Arsenal der zur Verfügung stehenden FV dieses Typs weit hinter den FV der anderen Typen zurück. Daher sei dieser Typ allenfalls zur Peripherie des Systems der FVG zu rechnen.

Seifert (2004: 70) weist ebenfalls auf das Bestehen von Kommutationsreihen („Reihenbildung“) auch bei nominativischen Verbindungen hin und sieht in dieser Eigenschaft offenbar ein Argument für die Zugehörigkeit der in Frage stehenden Fügungen zu den FVG. Unklar bleibt hierbei jedoch, weshalb bei Seifert z. B. erfolgen, geschehen, stattfinden als FV gelten, während Verben wie eintreten, die $\mathrm{zu}$ einer modifizierenden Reihenbildung beitragen, weiterhin ausdrücklich als Vollverben behandelt werden. Das ergibt dann folgende Opposition:

(25) a. Das Ereignis findet statt. [= durativ, FV]

b. Das Ereignis tritt ein. [= inchoativ, Vollverb]

Neben dem Bestehen von Kommutationsreihen erkennt Seifert (2004: 70) jedoch vor allem in einer anderen Eigenschaft eine Gemeinsamkeit zwischen FVG und nominativischen Fügungen mit Verbalabstraktum: Zwar liege ein wesentlicher Unterschied darin, dass das nomen actionis bei erfolgen nicht als Teil des Prädikatsausdrucks fungiere. Mit den FVG bestehe aber „eine ,Familienähnlichkeit“ insofern, als der prädikative Kern durch ein Verbalabstraktum realisiert wird“ (ebd.). Es fällt für Beispiele wie die in (25) allerdings schwer, den ,prädikativen

33 Helbig (1984) fasst auch deadjektivische Bildungen wie Uneinigkeit als nominale Komponente eines FVG auf. 
Kern' in etwas anderem als in den Prädikaten findet statt, tritt ein selbst zu verorten - das Subjekt hat hier jedenfalls keine prädikativen Eigenschaften. Die behauptete Familienähnlichkeit zwischen FVG und Konstruktionen mit stattfinden, erfolgen $\mathrm{u}$. ä. bleibt folglich wohl doch $\mathrm{zu}$ vage, als dass hier eine weitere Untergruppe der FVG anzusetzen wäre.

\subsection{Testverfahren zur Ermittlung von FVG}

\subsubsection{FVG und „Grundverb“}

Das Bemühen der Forschung, aufgrund bestimmter semantischer und syntaktischer Eigenschaften eine Klasse FVG zu begründen, war von Beginn an von dem Versuch begleitet, Testverfahren zu entwickeln, die den Ansatz eines FVG operationalisierbar machen sollten. Ein wesentlicher Test für den Ansatz eines FVG avant la lettre ist bereits durch die Sprachkritik vorgegeben: FVG sind ihr zufolge überflüssige „Schwell-“ oder „Streckformen“ zu einem etymologisch verwandten und gleichbedeutenden einfachen Verb (zur Aufführung bringen vs. aufführen, entscheiden vs. zur Entscheidung bringen). Auch in der frühen Forschung zu FVG wird auf die Ersetzbarkeit der Fügung durch ein Verb hingewiesen (Daniels 1963: 15; vgl. dazu auch Storrer 2006: 147).

von Polenz (1963: 26f.) hat für das einfache Verb, das der nominalen Komponente des FVG zugrunde liegt und das an die Stelle des gesamten FVG treten kann, den Begriff des „Grundverbs“ geprägt. Wie das Verhältnis zwischen Grundverb und FVG im Einzelnen beschaffen sein soll, wird bei von Polenz (1963) jedoch weitgehend unbestimmt gelassen: Während z. B. bei aufführen vs. zur Aufführung bringen noch Quasi-Synonymie zwischen Substantiv und einfachem Verb vorliegt, besteht bei zum Lachen bringen - nach von Polenz (1963: 14) ein kausatives FVG zum „Grundverb“ lachen - keine semantische Äquivalenz des gesamten FVG mit dem Verb. Als Grundverben können nach von Polenz (1963: 17) aber auch solche Verben gelten, die keine morphologische Beziehung zum Substantiv des FVG aufweisen: in Abrede stellen stehe als FVG in Relation zu abstreiten (leugnen), da der Zusammenhang mit abreden dem zeitgenössischen Sprecherbewusstsein nicht mehr zugänglich sei. Ein solches „Suppletivverhältnis“ (ebd.) wird etwa auch für in Angriff nehmen vs. anfangen, in Frage stellen vs. bezweifeln angenommen.

Indem von Polenz das Bestehen eines synonymischen und etymologisch verwandten Verbs nicht als maßgeblich für den Ansatz eines FVG ansieht, löst er sich deutlich von dem durch die Sprachkritik vorgegebenen Konzept der „Streck“ oder „Schwellform“, das ja stets die Existenz eines parallelen Verbs voraussetzt. 
Ähnlich wie bei von Polenz (1963) wird auch in der jüngeren Forschungsgeschichte die Existenz eines an die Stelle des FVG einsetzbaren stammgleichen Verbs zwar als Charakteristikum akzeptiert, nur selten jedoch als ,hartes' Kriterium für die Ermittlung eines FVG angesehen. ${ }^{34}$ So warnt Heringer (1968) ausdrücklich vor einer Überbewertung der Ersetzbarkeit durch ein einfaches Verb:

Allerdings darf man gerade die Möglichkeit des Ersatzes durch ein einfaches Verbum nur mit Vorsicht anführen. Jedenfalls bewegen wir uns damit im rein Zufälligen, da ja ein entsprechendes Verbum nicht zu existieren braucht.

(Heringer 1968: 29, Fußnote 18) $)^{35}$

Auch Helbig (1984: 168) führt die Ersetzbarkeit durch ein stammidentisches Vollverb zwar unter den „operationellen Kriterien“ für die Ermittlung von FVG, misst diesem Faktor jedoch offenbar nur begrenzte Gültigkeit bei: „Das FVG kann in den meisten Fällen durch das entsprechende Vollverb (bzw. Kopula + Adjektiv) substituiert werden (ohne völlige Bedeutungsidentität)“ [Hervorhebungen im Original]. In der jüngeren Forschung dient das Vorhandensein eines Grundverbs allenfalls noch als heuristisches Mittel zur Auffindung von FVG (vgl. von Polenz 1987: 174; Seifert 2004: 63), keinesfalls aber als deren notwendige oder hinreichende Eigenschaft. ${ }^{36}$

\subsubsection{Grammatische Proben}

Da die Ersetzbarkeit durch ein einfaches stammidentisches Verb kein sicheres Kriterium für den Ansatz eines FVG bot, wurde nach anderen (vorwiegend distributionellen) Verfahren gesucht, die eine Eingrenzung der FVG gewährleisten können. Im Folgenden sollen zunächst die gängigsten Testverfahren kurz vorgestellt werden; im daran anschließenden Abschnitt 2.5 werden sie dann auf ihre Tauglichkeit hin überprüft.

34 Lediglich Burger (1973: 40), Guttmacher (1980: 49, 70) und Burger/Buhofer/Sialm (1982: 37) scheinen diesem Kriterium eine erhöhte Bedeutung beizumessen.

35 Ähnlich vorsichtig äußert sich auch Engelen (1968: 291).

36 Angesichts der allgemeinen Zurückhaltung gegenüber dem Kriterium der Ersetzbarkeit durch ein einfaches Verb ist es nicht gerechtfertigt, der FVG-Forschung „eine Übergeneralisierung“ dieses Kriteriums vorzuwerfen, wie es Eisenberg (2006a: 312) tut. 


\subsubsection{Weglassproben}

Die nominale Komponente eines FVG kann nicht weggelassen werden, ohne dass sich die Lesart des Verbs ändert wie in (26a) oder dass eine ungrammatische Äußerung entsteht wie in (26b) (vgl. Engelen 1968: 289; Herrlitz 1973: 14f.; Helbig 1984: 172).

(26) a. Das Bild kommt zur Versteigerung. - \#Das Bild kommt. (nur lokal) ${ }^{37}$

b. Der Lehrer erteilt dem Schüler eine Rüge. - *Der Lehrer erteilt.

Das FV und das homonyme Vollverb sind einander „semantisch entfremdet“ (von Polenz 1987: 172); die Auslassung des FV bzw. Vollverbs in einem koordinierten Satz bildet daher ein Zeugma (vgl. Daniels 1963: 22f.; von Polenz 1987: 172; Seifert 2004: 59):

(27) a. *Sie brachte den Patienten ins Bett und zum Schweigen.

b. `Er kam ins Haus und ins Reden.

\subsubsection{Erweiterungsproben}

Das nomen actionis ist nur eingeschränkt durch Attribute, d. h. Adjektivattribute wie in (28a) oder attributive Relativsätze wie in (28b), erweiterbar (vgl. Engelen 1968: 292; Heringer 1968: 49; Helbig 1984: 170f.):

(28) a. 'Er brachte das Stück zur prachtvollen Aufführung.

b. 'Er brachte das Stück zu der Aufführung, die diesem gerecht wurde.

Allerdings wird etwa bei Engelen (1968: 292) darauf hingewiesen, dass bei FVG wie in (29), die noch nicht lexikalisiert seien, eine Attribuierung möglich sei:

(29) a. Er geriet in große Not.

b. Er stellte es ihm zur persönlichen Verfügung.

c. Man stellte ihn unter scharfen Arrest.

Weshalb aber gerade diese Fügungen nicht lexikalisiert sein sollen, bleibt fraglich; sie sind jedenfalls nicht weniger konventionalisiert als geläufige FVG wie zur Aufführung bringen oder Angst haben.

37 Wenn eine Äußerung eine nicht einschlägige Lesart enthält, wird dies durch „\#“ markiert. 


\subsubsection{Umstellproben}

Im Gegensatz zu Verbindungen mit einem Vollverb kann das FV und der nominale Teil eines FVG im Nebensatz nicht getrennt werden, z. B. durch sofort wie in (30) oder nicht wie in (31) (Heringer 1968: 48f.; Herrlitz 1973: 13; Helbig 1984: 171): $:^{38}$

(30) a. Der Regisseur will, dass das Stück sofort zur Aufführung kommt.

b. ${ }^{\star}$ Der Regisseur will, dass das Stück zur Aufführung sofort kommt.

a. Der Regisseur will, dass das Stück sofort zur Aufführung kommt.

b. *Der Regisseur will, dass das Stück zur Aufführung nicht kommt.

Engelen (1968: 291) nimmt eine weitere Stellungseigenschaft von FVG an: „Wird [...] ein Satz mit Funktionsverbgefüge um eine statische Ortsangabe erweitert, so ist die Gliedfolge von Ortsangabe und nominalem Teil des Funktionsverbgefüges vollkommen fest". So bestehe für Ortsangaben bei Vollverben die Möglichkeit eines Positionswechsels, vgl. (32), die bei Funktionsverben nicht möglich sei, vgl. (33):

(32) a. Seine Erzeugnisse standen zum Verkauf auf dem Markt.

b. Seine Erzeugnisse standen auf dem Markt zum Verkauf.

a. Diese Mittel standen ihm in Frankfurt zur Verfügung.

b. *Diese Mittel standen ihm zur Verfügung in Frankfurt.

(Engelen 1968: 291)

38 Als dem zuwiderlaufende Stellungseigenschaft von FVG wird bei Daniels (1963: 24) auch die „Klammerfähigkeit“ genannt, die sogar als „,sicheres Kriterium“ bezeichnet wird (vgl. auch Relleke 1974: 8; Yuan 1982: 9). „Klammerfähigkeit“ zeigt z. B. der folgenden Satz: Wird eine abgetretene Forderung [...] nochmals an einen Dritten abgetreten, so finden, wenn der Schuldner an den Dritten leistet [...], zugunsten des Schuldners die Vorschriften des $\S 407$ dem früheren Erwerber gegenüber entsprechende Anwendung (Beispiel aus Daniels ebd.). Heringer (1968: 42, Fn. 58) hat jedoch darauf hingewiesen, dass gerade die Verbindung eines Verbs mit adverbialer Bestimmung ebenfalls Klammerbildung zeigt und dass folglich von einem „sicheren Kriterium“ keine Rede sein könne: Der Bäcker bringt die Brötchen morgens gegen fünf Uhr in den Lieferwagen. Kritisch zur Klammerfähigkeit auch Herrlitz (1973: 13); Persson (1975: 12); van Pottelberge (2001: 251-253). 


\subsubsection{Paraphraseproben}

Engelen (1968: 292) geht davon aus, dass der „nominale Teil eines Funktionsverbgefüges nicht in ein Präpositionaladverb mit einem Gliedsatz auflösbar“ sei (vgl. auch Persson 1992: 158):

(34) a. Er brachte diesen Fall zum Abschluss.

b. `Er brachte diesen Fall dazu, abgeschlossen zu werden.

Dementsprechend schließt dieser Test laut Engelen (1968) allerdings auch die kausativen bringen-Fügungen, die seit Kolb (1962), Daniels (1963) und von Polenz (1963) allgemein als FVG gelten, aus der Klasse der FVG aus:

(35) a. Er brachte Fritz zum Nachgeben.

b. Er brachte Fritz dazu, nachzugeben.

\subsubsection{Ersatzproben}

FV stellen eine geschlossene Klasse dar, d. h. sie sind nicht oder nur sehr begrenzt durch andere Verben ersetzbar (Engelen 1968: 293ff.; Heringer 1968: 44; Herrlitz 1973: 17f.; Helbig 1984: 169; von Polenz 1987: 175f.). Vollverben hingegen sind Elemente einer umfangreichen paradigmatischen Liste:

(36) a. Das Buch geht/^läuft/*rennt heute in den Druck.

b. Der Junge geht/läuft/rennt zum Bahnhof.

\subsubsection{Erfragbarkeit/Anaphorisierbarkeit}

Der nominale Teil eines FVG kann im Gegensatz zu den Angaben eines Vollverbs nicht erfragt werden (Engelen 1968: 290; Helbig 1984: 170), vgl. hier das FVG (37a) gegenüber dem Vollverb in (37b):

(37) a. Das Stück kommt zur Aufführung. - *Wohin/ ${ }^{\star}$ wozu kommt das Stück?

b. Das Kind kommt zum Theater. - Wohin kommt das Kind?

Das Substantiv der Umschreibung ist auch nicht anaphorisierbar, d. h. es kann nicht durch ein Pronomen oder ein Pronominaladverb wiederaufgenommen werden (Daniels 1963: 23f.; Engelen 1968: 290; Heringer 1968: 48; Herrlitz 1973: 19; Helbig 1984: 169): 
(38) a. Das Bild kam zur Versteigerung.

b. ${ }^{\star}$ Dazu kam das Bild.

\subsubsection{Negation}

Ein FVG wird vorzugsweise nicht durch kein (Wortnegation), sondern durch nicht (Satznegation) verneint. Dies gilt zumindest für FVG, die eine PP enthalten, s. dazu (39). FVG mit nomen actionis im Akkusativ können jedoch, falls ein Nullartikel steht, mit nicht oder kein negiert werden, vgl. (40) (zu diesem Test Heringer 1968: 48f.; Herrlitz 1973: 13; Helbig 1984: 171).

(39) a. nicht zum Stehen kommen/bringen- ${ }^{\star}$ zu keinem Stehen kommen/bringen

b. nicht zur Anwendung kommen/bringen - ?zu keiner Anwendung kommen/bringen

(40) a. Er leistet der Aufforderung nicht/keine Folge.

b. Er nahm auf seine Freunde nicht/keine Rücksicht.

(Beispiele Helbig 1984: 171)

Dennoch kann nach Heringer (1968: 49f.) gelegentlich auch bei präpositionalen FVG eine kein-Negation vorliegen. Ein solcher Fall „setzt aber eigentlich die Auflösung der Funktionsverbfügung voraus“ (49):

(41) a. Das Buch kam zu keinem Abschluss.

b. Sie steht in keiner Beziehung zu uns.

\subsubsection{Passivbildung}

Die Möglichkeit der Passivbildung und damit die Subjektfähigkeit des nominalen Teils von FVG ist eingeschränkt (Helbig 1984: 170):

(42) a. Die Herstellungstechnik erfuhr eine Vereinfachung.

b. `Eine Vereinfachung wurde von der Herstellungstechnik erfahren.

c. Die Soldaten nahmen Aufstellung.

d. *Aufstellung wurde von den Soldaten genommen. 


\subsection{Zur Leistungsfähigkeit der Testverfahren}

Lässt man das vorgestellte Inventar an Testverfahren Revue passieren, so ist zunächst festzuhalten, dass die Autoren selbst vielfach einräumen, dass einzelne Tests keine konsistenten Ergebnisse liefern. So bietet Daniels (1963: 23) einige Belege dafür, dass - entgegen dem von ihm selbst postulierten Kriterium der fehlenden Erweiterungsmöglichkeit (hier Kriterium b) - gelegentlich auch Relativsätze an Nomina von FVG angeschlossen werden könnten, vgl. (43):

Als er den Wert, den ich auf diese Skizze legte, gesehen, hat er sie mir gerne geschenkt. (A. v. Chamisso)

Auch Helbig (1984) gesteht zu, dass die vorgeschlagenen Kriterien durchaus ihre Grenzen haben. Obwohl laut Helbig (1984: 173) die mangelnde Erfragbarkeit und Pronominalisierung „zu den eindeutigsten Kriterien gehören“, gebe es unter den FVG Fälle, in denen sehr wohl Erfragbarkeit und Pronominalisierung möglich sei. Dabei handelt es sich vorwiegend um FVG mit einem Nomen im Akkusativ:

(44) a. Er nimmt die Verhandlungen mit dem Nachbarstaat auf.

b. Er nimmt sie auf.

c. Was nimmt er auf?

(Beispiele Helbig 1984: 173)

Bei Helbig (1984: 174) wird gleichfalls eingeräumt, dass auch die Unmöglichkeit der Attribuierung kein absolut gültiges Kriterium sei, wie bereits Engelen (1968: 292) festgestellt hatte. Dies zeige sich etwa darin, dass einige Nomina in FVG sogar obligatorisch ein Attribut fordern:

(45) a. *Die Versammlung nahm einen Verlauf.

b. Die Versammlung nahm einen ausgezeichneten Verlauf.

Andere Tests, die von den Autoren selbst nicht relativiert werden, erweisen sich schon bei oberflächlicher Betrachtung als unergiebig. So kann von einer fehlenden Passivbildung - Kriterium h) - gerade für den allgemein als zentral angesehenen präpositionalen Typus nicht die Rede sein:

(46) a. Der Regisseur bringt das Stück zur Aufführung.

b. Das Stück wird durch den Regisseur zur Aufführung gebracht. 
Auch die von Engelen (1968: 291) vorgeschlagene Regel, der zufolge eine Ortsangabe in einem Satz mit FVG nicht umgestellt werden könne, s. o. Test c), erweist sich als nicht zielführend. So ist der von Engelen als ungrammatisch bewertete Satz (33b) mit Kontrastbetonung oder in Aufzählungen sehr wohl akzeptabel:

(47) a. Diese Mittel standen ihm zur Verfügung in Frankfurt, keine anderen.

b. Diese Mittel standen ihm zur Verfügung in Frankfurt, nicht aber in Offenbach.

c. Diese Mittel standen ihm zur Verfügung in Frankfurt, Offenbach und München.

Andere Tests - etwa der Zeugmatest, Ersatz- und Paraphraseproben, die spezifischen Negationseigenschaften - kommen auf den ersten Blick zu konsistenteren Ergebnissen (zur Negation vgl. allerdings Kap. 6.6). Eine Lösung des Abgrenzungsproblems könnte folglich schlichtweg darin bestehen, die untauglichen Tests auszuschließen und die Menge der einschlägigen Testverfahren auf die zuletzt genannten zu begrenzen. Dabei stellt sich jedoch die grundsätzliche Frage, welche Eigenschaften von FVG mit den genannten Tests überhaupt ermittelt werden sollen. Auf die Funktionen von FVG, die allgemein als entscheidendes Merkmal der Klasse angesehen werden (Aktionsartenmarkierung, Kausativ- und Passivumschreibung), bezieht sich jedenfalls keiner der vorgestellten Tests. Die genannten Verfahren belegen im Großen und Ganzen nur, dass es sich bei FVG offenbar nicht um freie Wortkombinationen, sondern in irgendeiner Weise um feste Verbindungen handelt (dazu weiter Kap. 4). Dies zeigt sich daran, dass die genannten Tests gleichermaßen auf Phraseologismen angewandt werden können. So gilt auch für Phraseologismen, dass die nominalen Komponenten nicht weglassbar, nicht erfragbar, nicht pronominalisierbar und nicht attribuierbar sind: ${ }^{39}$

(48) a. Er warf die Flinte ins Korn. - \#Er warf die Flinte.

b. Er warf die Flinte ins Korn. - \#Wohin warf er die Flinte?

c. Er warf die Flinte ins Korn. - \#Sie war groß.

d. Er warf die Flinte ins Korn. - \#Er warf die Flinte ins Korn des Bauern.

$39 \mathrm{Zu}$ diesen Merkmalen, die wesentlich die Festigkeit von Phraseologismen ausmachen, vgl. Burger (2010: 15-29). 
Dass der nominale Bestandteil eines FVG nicht in einen Nebensatz umgewandelt werden kann, s. o. Test d), ist ebenfalls keine besondere Eigenschaft von FVG. In diese Beschränkung kommt lediglich ein ganz allgemeines Merkmal von festen Phraseologismen zum Tragen, nämlich dass syntaktische Variation nicht oder nur stark eingeschränkt möglich ist (Burger 2010: 21-23), vgl. das klassische Beispiel (49):

(49) Das ist kalter Kaffee. - \#Das ist Kaffee, der kalt ist.

Auch die Zeugmabildung findet sich bei Phraseologismen ebenso wie bei FVG:

(50) a. \#Er warf die Flinte ins Korn und die Schaufel auf den Boden.

b. \#Schneewittchen biss in den Apfel und dann ins Gras.

Die bei FVG mit PP weitgehend fehlende Möglichkeit einer Negation mit kein kennzeichnet ebenfalls eine ganze Reihe von Phraseologismen:

(51) a. nicht ins Gras beißen - \#in kein Gras beißen

b. nicht die Flinte ins Korn werfen - \#keine Flinte ins Korn werfen

Die Gründe für das angesprochene Negationsverhalten von Phraseologismen und FVG sind allerdings weniger in der Negation selbst als vielmehr beim nicht negierten Artikel zu suchen: Da bei präpositionalen FVG meist kein unbestimmter Artikel steht, tritt dementsprechend das mit dem unbestimmten Artikel ein korrelierende kein nicht auf (vgl. Heringer 1968: 48-50; s. auch Kap. 6.6). Auch bei Phraseologismen, die keinen unbestimmten Artikel enthalten, fehlt in der Negation das entsprechende kein - da *in ein Gras beißen als Phraseologismus nicht existiert, ist wenig überraschend, dass auch entsprechendes *in kein Gras beißen ungrammatisch ist; Wendungen mit ein dagegen können sehr wohl kein-Negation enthalten (z. B. ein Gesicht wie drei Tage Regenwetter machen - kein Gesicht wie drei Tage Regenwetter machen).

$\mathrm{Zu}$ den Tests für FVG bleibt somit festzuhalten, dass bisher kein operationelles Verfahren gefunden werden konnte, das FVG eindeutig identifizierbar macht. Die vorgestellten Verfahren leisten vor allem keine Abgrenzung von der Gruppe 
der Phraseologismen. ${ }^{40}$ Ferner ist, wie oben bereits ausgeführt, unklar, auf welche Eigenschaften der Klasse FVG sich die vorgestellten Tests überhaupt beziehen sollen. Für die bisher postulierten Kernmerkmale der Klasse - Aktionsartenmarkierung, Kausativ- und Passivumschreibung - sind diese Tests jedenfalls nicht einschlägig.

\subsection{Light verbs - verbes support: FVG-ähnliche Strukturen in anderen Sprachen}

\subsubsection{FV und FVG in der anglistischen Forschungstradition}

Nomen-Verb-Verbindungen, die mit den als FVG bezeichneten Konstruktionen des Deutschen vergleichbar sind, gibt es auch in vielen anderen europäischen und außereuropäischen Sprachen. Während sich in der germanistischen Forschung der Begriff FVG weitgehend durchgesetzt hat, verfügt laut van Pottelberge (2007: 436) vor allem die anglistische Sprachwissenschaft über eine relativ große Bandbreite von Termini. Die Vielfalt der Begriffe geht dabei allerdings, so van Pottelberge, weniger auf abweichende Gegenstandsbestimmungen zurück, sondern reflektiert eher je eigene Forschungstraditionen mit ihren spezifischen terminologischen Festlegungen (van Pottelberge 2007: 436). Im Einzelnen werden die folgenden Begriffe verwendet:

- „light verb“, „light verb construction“ (Jespersen 1942: 117; Grimshaw/Mester 1988; Brugman 2001: 551; Hale/Keyser 2002: 21; Butt 2010: 48),

- „composite predicate“ (Cattell 1984; Hinrichs/Kathol/Nakazawa 1998; Akimoto/Brinton 1999: 21),

- „expanded predicate“ (Algeo 1995: 203),

- „thin verb“ (Allerton 2001: 7),

- „stretched verb construction“ (Allerton 2001: 6),

- „support verb constructions“ (Ronan 2012: 9).

Anders als in der germanistischen Forschung ist in der Literatur zum Englischen das Kriterium eines funktionalen Mehrwerts offenbar nicht maßgeblich für den Ansatz der Klasse. Als ausschlaggebend werden hier stattdessen meist strukturelle Eigenschaften der betreffenden Nomen-Verb-Verbindungen genannt: „In

40 Die Forschung hat zwar keine brauchbaren Testverfahren für eine solche Abgrenzung entwickelt, jedoch durchaus Überlegungen angestellt, wie das Verhältnis der FVG zur Klasse der festen Verbindungen zu beurteilen ist. Vgl. dazu die Diskussion in Kap. 4. 
English linguistics, the common ground is that the structures should be non-compositional and consist of a semantically low-content, inflected verb and a predicate noun“ (Ronan 2012: 9). Mit der Festlegung auf einen nicht-kompositionalen Aufbau wird der Tatsache Rechnung getragen, dass zwei Ereignisversprachlichungen, d. h. das Verb und das ,predicative noun' nur ein einziges Ereignis bezeichnen; die Fügung kann daher nicht als reguläre Verknüpfung zweier Ausdrücke verstanden werden, deren jeweilige Bedeutungen die Gesamtbedeutung der Fügung ergeben. Die anderen in der zitierten Definition verwendeten Begriffe lassen allerdings erheblichen Interpretationsspielraum: So wäre zu klären, was genau unter einem ,semantically low-content verb‘ zu verstehen ist. Wie inhaltsarm muss das Verb sein, um als ,light verb“ (o. ä.) gelten zu können? ${ }^{41}$ Die semantische Bandbreite der hier vorkommenden Verben ist jedenfalls beträchtlich: So werden neben have und be (z. B. Allerton 2001: 7, 207), die zweifelsohne eine relativ abstrakte Bedeutung aufweisen, auch Verben wie make, give, take (Quirk et al. 1985: 750-752; Wierzbicka 1982; Allerton 2001: 207) sowie demgegenüber relativ merkmalreich erscheinende Verben wie conduct, indulge in oder burst into genannt (Allerton 2001: 207).

Besonders umstritten ist die Bestimmung der in der Konstruktion möglichen Nomina. Hinter dem Minimalkonsens, dass es sich um ein ,predicative noun“ handeln müsse, tun sich beträchtliche Unschärfen auf: Autoren wie Wierzbicka (1982) und Stein (1991) akzeptieren lediglich Nullableitungen von Verben, wie sie in have a walk oder take a look vorliegen, als Elemente der Konstruktion. Quirk et al. (1985: 750-752) und Algeo (1995: 206) erweitern dagegen den Bestand der in Frage kommenden Nomina um alle deverbalen Nomina, und zum Teil werden sogar generell nicht-wörtlich $\mathrm{zu}$ verstehende Kombinationen von Verben wie take oder make mit Substantiven (take place, make way) zum erweiterten Kreis der light-verb-Konstruktion gerechnet (vgl. Ronan 2012: 11).

Der Blick auf die anglistische Forschung zeigt somit, dass auch eine andere Ausrichtung der definitorischen Versuche, die weniger auf die Funktion als vielmehr auf die Struktur und die Beschaffenheit von Nomen und Verb abzielt, keine befriedigende Lösung bietet. Das Hauptproblem liegt darin, dass keine objektivierbaren Entscheidungskriterien dafür herausgearbeitet werden, weshalb ein bestimmtes Nomen oder Verb als Element einer light-verb-Konstruktion gelten soll und ein anderes nicht. Mangels einer solchen Entscheidungsbasis sind hier nur mehr oder weniger willkürliche Setzungen möglich.

41 Zur beträchtlichen Unschärfe von Begriffen wie, light verb` vgl. auch van Pottelberge (2001: 72). 


\subsubsection{FV und FVG in der romanistischen Forschungstradition}

Im Gegensatz zu den FVG des Deutschen und teilweise auch des Englischen (Brinton 1996: 189-193) sind die entsprechenden Nomen-Verb-Verbindungen der romanischen Sprachen nur selten Gegenstand sprachkritischer Auseinandersetzungen gewesen (vgl. Heringer 1968: 108-110 zur französischen Stilistik). Ein Grund für den etwas entspannteren Umgang mit diesen Fügungen in der Romanistik ist wohl darin zu sehen, dass Nomen-Verb-Verbindungen - zumindest wenn man auf das Französische blickt - wesentlich häufiger als im Deutschen zu sein scheinen (Kotschi/Detges/Cortès 2009: X). Im Hinblick auf die Frage nach einer genauen Abgrenzung dieser Konstruktionsklasse hat sich in der romanistischen Sprachwissenschaft vor allem das Konzept des „verbe support“ als einflussreich erwiesen, das der Theorietradition der von M. Gross (1975, 1980) begründeten „lexique-grammaire“ entstammt. In dieser an Z. Harris anschließenden transformationellen Grammatiktheorie werden Sätze grundsätzlich als lexikalische Aktualisierungen eines elementaren Schemas begriffen (zum Modell vgl. die Skizze in van Pottelberge 2001: 154-159). Die verbes support spielen in der „lexique-grammaire“ insofern eine wichtige Rolle, als sie bei der Aktualisierung von nominalen Prädikaten zum Tragen kommen: „la fonction d'un verbe support est d'actualiser un prédicat nominal“ (G. Gross 1993: 16). Da dessen Funktion lediglich in der syntaktischen Realisierung eines nominalen Prädikats besteht, kann ein solches Verb keine eigene prädikative Bedeutung tragen - in avoir plaisir ist avoir somit als semantisch leer und lediglich als Träger der grammatischen Informationen zu Person, Numerus und Tem-pus zu sehen: „[...] la fonction prédicative est portée par le substantif $\mathrm{N}$ et ses compléments éventuels, le verbe n'étant que le support des marques de temps et de personne“ (Vivès 2004: 641). Als Mittel der syntaktischen Realisierung erinnern die verbes support somit an den semantisch leeren Operator der ,lexikalischen Funktionslehre‘ von Mel'cuk (1996).

Insofern, als lediglich Prädikate wie avoir in avoir peur als verbes support gefasst werden, ist der Begriff klarer abgegrenzt als der des Funktionsverbs oder des light verbs, die ja, wie gezeigt, ein sehr breites Spektrum von semantisch mehr oder weniger ,leeren' Verben umfassen und deshalb wesentlich unschärfer sind. Ob avoir aber tatsächlich lexikalisch-semantisch leer ist und seine Funktion allein auf die Aktualisierung des prädikativen Nomens beschränkt ist, bliebe noch im Einzelnen zu zeigen.

Allerdings wird auch in der lexique-grammaire der Begriff des verbe support beträchtlich ausgedehnt. So wird eine große Zahl von merkmalreicheren Verben, die in irgendeiner Weise als semantische Erweiterungen eines einfachen verbe 
support gesehen werden können, ebenfalls unter diesem Begriff gefasst, und zwar als „verbe support variantes“ gegenüber den „verbes support standard“, vgl. G. Gross (1989, 169-182). Auf diese Weise werden z. B. neben donner in der Kombination donner une invitation auch die Verben in recevoir une invitation, accorder une invitation sowie renouveler une invitation als verbes support im erweiterten Sinne verstanden (vgl. G. Gross 1989: 180). Gerade diese Erweiterung lässt aber auch die Frage aufkommen, wie die Klasse der verbes support beschränkt werden kann. Um zu klären, ob etwa retirer une invitation (eine Einladung zurücknehmen') ein verbe support zumindest in diesem erweiterten Sinne sein kann, müssten die Kriterien der Ableitung der „verbes supports variantes“ aus den „verbes support standard“ wesentlich detaillierter beschrieben sein. Eine Beschränkung, die bloß so weit geht, dass verbes support durch die Verbindung mit einem prädikativen Nomen definiert sind (so Giry-Schneider 1987: 19, 27-32), wird kaum ausreichen. Wenn im Prinzip jedes Verb, das mit einem prädikativen Nomen kombiniert ist, als verbe support in Frage kommt, ist ein eigener Begriff hierfür letztlich überflüssig - ,Verb mit nomen actionis` o. ä. wäre dann als Bestimmung völlig ausreichend.

\subsubsection{FV und FVG in außereuropäischen Sprachen}

FVG-ähnliche Verbindungen von Nomen und Verb kommen nicht nur in den Sprachen Europas vor, wie die Beispiele in (52) zeigen, sondern sie sind in sehr vielen außereuropäischen Sprachen verbreitet, vgl. (53).

(52) a. lat. veniam dare ,Erlaubnis geben“

b. frz. donner une réponse ,Antwort geben“

c. engl. give an answer

d. nl. antwoord geven

е. russ. приказ дать ,Befehl geben“/,befehlen‘ (RDW 140)

(53) a. apan. keikoku suru ,Warnung + machen' (Grimshaw/Mester 1988: 212)

b. chin. geiyi bangzhù ,geben + Hilfe‘ (Yuang 1983: 194)

c. pers. fekr kærdæn ,Gedanke + tun`/,denken“ (Family 2014: 214)

d. türk. park etmek ,Parken + machen'/,parken', selam vermek ,Gruß geben'/,grüßen“ (Ströbel 2008: 117)

e. inguschisch noq'uostal dar ,Hilfe + tun` (Koptjevskaja-Tamm 1993: 281)

f. Telegu (Dravidisch) prayanamu-nu cesidi ,Reise tat‘ (Jäger 2006: 71) 
Wie kaum anders zu erwarten, macht die Vielzahl der Sprachen und einzelphilologischen Beschreibungstraditionen es schwer, einen gemeinsamen begrifflichen Nenner zu finden. Festgehalten werden kann aber immerhin eine sehr verbreitete Verwendung des auf Jespersen (1942) zurückgehenden Terminus „light verb“/,light verb construction“ (s. o. Abschnitt 2.6.1). Im Unterschied zu Jespersen und auch zu der germanistischen Forschungstradition werden light verbs allerdings nicht nur für $\mathrm{N}+\mathrm{V}$-Verbindungen, sondern zum Teil auch für V+V-Kombinationen angesetzt (vgl. Butt 2003: 2; Baker/Harvey 2010: 14); damit überschneidet sich der Begriff mit dem des ,seriellen Verbs' (dazu Aikhenvald 2006: 1). ${ }^{42}$ Als Beispiel für eine V+V-Kombination mit dem als light verb klassifizierten ,geben'-Verb aus dem Urdu vgl. (54).

\section{(54) naadyaa=ne makaan banaadii-yaa. \\ Nadya.f.sg.=erg house.m.nom make give-perf.m.sg \\ „Nadya built a house (completely, for somebody else).“}

(Butt 2010: 52)

Richtungweisende Ansätze für die Entwicklung eines sprachenübergreifenden light-verb-Begriffs hat Butt (2003: 3f., 2010: 72) vorgelegt. Sie hält fest, dass light verbs nur gemeinsam mit einem „main verb“ eine Prädikation bildeten: „[...] they hook onto another event predication and deploy their lexical content dependently“ (Butt 2010: 73). Das zentrale Merkmal sieht sie jedoch in den Funktionen, die „light verbs“ in Bezug auf die durch das main verb ausgedrückte Ereignisprädikation übernehmen: „The function of light verbs is to modulate the event predication of a main predicator in the clause“ (2010: 74). Die jeweilige Modulationsleistung kann dabei je nach Sprache sehr unterschiedlich ausfallen: „This modulation can be seen in terms of providing more information about the typical parts of an event: who did the causation, what the result was, whether the event was bounded or whether it was benefactive, sudden, agentive/volitional, accidental, etc.“ (ebd.). Butts Beispiele zeigen aber, dass die postulierte Modulationsleistung häufig erst durch Oppositionspaare, zu denen sich einzelne light verbs

42 „A serial verb construction (SVC) is a sequence of verbs which act together as a single predicate, without any overt marker of coordination, subordination, or syntactic dependency of any other sort. Serial verb constructions describe what is conceptualized as a single event" (Aikhenvald 2006: 1). Der entscheidende Unterschied zwischen seriellen Verben und „light verbs“ bzw. Funktionsverben ist in der syntaktischen Markierung von Koordination bzw. Subordination zu sehen. Eine solche Markierung fehlt bei seriellen Verben im Gegensatz zu den „light verbs“ bzw. Funktionsverben. 
zusammenschließen, zustande kommt. So hängt etwa die Perspektivierung des Ereignisses als accidental bzw. agentive/volitional in den Urdu-Beispielen (55)(56) an dem Gegensatz zwischen dem ,tun'-Verb und dem ,kommen'-Verb.

naadya=ne kahaanii yaad $k$-ii.

Nadya.f.sg.=erg story.f.sg memory.f do-perf.f.sg

„Nadya remembered the story.“

(56)

naadya $=k o \quad$ kahaanii yaad aa-yii.

Nadya.F.SG=DAT story.F.SG memory.F come-PERF.F.SG

„Nadya remembered the story (the memory of the story came to Nadya).

(Butt 2010: 52)

Entscheidend für die Differenzierung des Ereignisses als „,accidental“ bzw. „,volitional“ dürfte in diesem Fall allerdings nicht allein der minimale Bedeutungsgehalt sein, der dem light verb zugesprochen wird (vgl. auch Butt/Lahiri 2013: 24), sondern schlicht der Kasusgegensatz Ergativ vs. Dativ. Welche Rolle solche Gegensatzpaare für die These einer bedeutungsmodulierenden Funktion der light verbs haben, wird im Vagen gelassen. So bleibt insbesondere unklar, worin die semantische Modulation bei Verben bestehen soll, die offensichtlich lediglich der syntaktischen Integration eines Prädikatsnomens dienen und die daher seitens der „lexique-grammaire“ nicht zu Unrecht als semantisch leere Prädikate aufgefasst werden (s. o. Abschnitt 2.3.1). Dass die semantische Modulation sehr vielfältig und zum Teil schwer greifbar sind, räumt Butt indes ein (,some of the semantic contributions are quite subtle“ 2010: 75); sie führt dies auf eine Eigenschaft der Verben selbst zurück: „The verbs which allow light verb readings have semantic specifications that are of a very general nature" (ebd.; vgl. auch Kap. 8.1.4).

An dieser Stelle ist festzuhalten, dass Butts Bestimmung des Begriffs light verb der germanistischen Forschungstradition in gewisser Hinsicht überraschend nahe steht, auch wenn sie selbst hier keinen direkten Bezug herstellt: In beiden Herangehensweisen wird das Vorhandensein einer Funktion als das zentrale Definitionselement angesehen (bei Butt ist dies die „modulation“), und in beiden Fällen bleibt der genaue Inhalt der postulierten Funktion relativ vage. Butts Definition weicht von dem germanistischen FVG-Begriff allerdings insofern deutlich ab, als sie gerade die Markierung von Aktionsarten nicht als Eigenschaft von light verbs ansieht, sondern diesen Zuständigkeitsbereich den Auxiliaren zuschlägt (Butt 2010: 71f.; Butt/Lahiri 2013: 9-11). Light verbs und Auxiliare werden explizit als getrennte syntaktische Klassen angesehen. Innerhalb des minimalistischen Beschreibungsrahmens würde dies bedeuten, dass light verbs in der vP/ 
VP enthalten sind, während Auxiliare, sofern sie Tempus und Aspekt/Aktionsart ausdrücken, in den entsprechenden übergeordneten Knoten AspP und TP zu lokalisieren sind (Argumente dafür anhand des Urdu bei Butt/Geuder 2001 und Butt/Lahiri 2013).

So schwierig die Bestimmung eines für mehrere Sprachen verwendbaren Konzepts ,light verb“/,Funktionsverb“,verbe support‘ auch ist - nicht zu Unrecht spricht Butt mehrfach vom „light verb jungle“ -, bleibt doch festzuhalten, dass die Klasse der Verben, die als Kandidaten für eine solche Kategorisierung in Frage kommen, über die einzelnen Sprachen hinweg offenbar erstaunlich konsistent ist. Ausdrücke mit einer entsprechenden Lesart sind laut Amberber (2010: 314) typischerweise Verben für ,sein', ,werden', ,kommen', ,tun‘, ,geben‘, ,gehen“, ,haben', ,machen‘, ,stellen', ,stehen“ und ,sagen'. Dass es sich dabei um Verben handelt, die auch in ihrer ,Vollverb'-Lesart eine verhältnismäßig schematische Bedeutung tragen bzw. grundlegende Sachverhalte von wahrscheinlich universaler Relevanz versprachlichen (,humanely relevant scenes“ Goldberg 1995: 39-41), scheint kein Zufall zu sein. Da sich auch die hier untersuchten FV bzw. FVG des Deutschen aus diesem möglicherweise universalen Kernbestand speisen, wird auf deren besondere Semantik und deren Rolle in N+V-Verbindungen des Deutschen noch einzugehen sein.

\subsection{Fazit}

Die Annahme, dass FVG spezifische semantische „Funktionen“ besitzen, zieht sich - bei allen strittigen Fragen und einander widersprechenden Ansichten - als roter Faden von den ersten sprachwissenschaftlichen Beiträgen zum Thema bis in die jüngere und jüngste Forschung. Dabei wurde neben der Kausativierung und der Passivumschreibung vor allem die Funktion der Aktionsartendifferenzierung als Hauptleistung der FVG herausgestellt. Seit Beginn der einschlägigen Forschung wurde Wert auf die Feststellung gelegt, dass die durch FVG ausgedrückten Funktionen durch einfache Verben nicht wiedergegeben werden könnten und dass FVG daher - entgegen den Anfeindungen der Sprachkritik - keine bloßen ,Schwell-‘ oder ,Streckformen' darstellten, sondern einen ,semantischen Mehrwert‘ besäßen. In der jüngeren Forschung wurde darüber hinaus vor allem der Systemcharakter der FVG betont: FV drückten durchgehend grammatische Bedeutungen - Aktionsart, Kausativ, Passiv - aus und seien daher mit den Hilfsverben vergleichbar. Die Systemhaftigkeit von FVG zeige sich nach allgemeiner Auffassung auch darin, dass FVG häufig Kommutationsreihen bildeten (in Bewegung kommen/bringen/sein). Das Vorhandensein einer systematisch zu beschreibenden Funktion und die Reihenbildung wird vor allem in der breit rezipierten 
Untersuchung von Polenz' (1987) als konstitutives Merkmal von FVG gesehen, mit deren Hilfe sich FVG von anderen Verb-Nomen-Verbindungen (bei von Polenz 1987 „Nominalisierungsverbgefüge“) angeblich abgrenzen lassen.

Bei näherer Betrachtung hat sich jedoch gezeigt, dass weder der systematisch beschreibbare Bedeutungsbeitrag noch die Reihenbildung notwendig und hinreichend für die Konstituierung einer eigenen Klasse FVG sind. Beide Eigenschaften gelten auch für Fügungen, die von ihrer syntaktischen Struktur her nicht mit FVG vergleichbar sind. So weisen Phraseologismen ebenfalls Verben auf, die eine Aktionsart markieren und in Kommutationsreihen stehen (aus dem Häuschen geraten/bringen/sein). Die systematische Beschreibbarkeit der FV ist auch dadurch in Frage gestellt, dass nicht nur eine geschlossene Klasse mit Elementen wie kommen, sein, bringen, sondern eine sehr große Zahl von Verben eine aktionsartensignalisierende Funktion übernehmen kann: in Jubel ausbrechen, in Verzweiflung verfallen, in Schwierigkeiten stecken usw. Angesichts der Vielzahl von Ausdrucksmöglichkeiten für eine Inhaltskategorie kann von einem System, das ja prinzipiell eine Eins-zu-eins-Entsprechung von Form und Inhalt voraussetzt, nicht die Rede sein.

Nicht haltbar ist ferner der immer wieder als klassenkonstituierendes Kriterium herangezogene, semantische Mehrwert'von FVG gegenüber einfachen Verben, der vor allem in der Signalisierung von Aktionsarten gesehen wird: So wird oftmals durch ein FVG (z. B. zur Verfügung stehen) keine andere Aktionsart zum Ausdruck gebracht als durch ein entsprechendes einfaches Verb (verfügen über). Das Beharren auf einem ,semantischen Mehrwert‘, einer besonderen Funktionalität von FVG gegenüber einfachen Verben zeigt letztlich auch, dass sich auch die neuere Forschung noch immer nicht von den Bewertungsmaßstäben der Sprachkritik befreit hat, denen zufolge ein sprachlicher Ausdruck ,wertvoll` zu sein habe.

In der jüngsten Forschung scheint sich die Einsicht, dass die Klasse der FVG nicht nach dem Vorhandensein eines eng begrenzten Sets von Funktionen bestimmt werden kann, mehr und mehr durchzusetzen. ${ }^{43}$ Die Annahme, dass FVG einen funktionellen Mehrwert besitzen, wird jedoch grundsätzlich nicht in Zweifel gezogen. So betont Seifert (2004: 72) zwar, dass es fraglich sei, „ob ein semantischer Mehrwert immer eindeutig zu bestimmen ist oder ob nicht auch mehrere Aspekte die Verwendung von FVG nahelegen“. Das grundsätzliche Gegebensein

43 Vgl. Eroms (2000: 167): „Wie bei den meisten periphrastischen Verbfügungen - und bei den verbalen Paradigmen allgemein - lässt sich bei den FVG nicht eine einzige und stets identische syntaktische oder semantische Funktion ermitteln“; in diesem Sinne auch Heine (2006: 44f.) und Seifert (2004: 12f.). 
eines solchen funktionellen Mehrwerts wird jedoch auch von Seifert nicht bestritten. Das Spektrum der möglichen Funktionen, das bei von Polenz (1987) noch sehr übersichtlich war, wird lediglich aufgefächert: FVG tragen laut Seifert (2004: 107) „eine Vielzahl potentieller satzsemantischer, syntaktischer und pragmatisch-stilistischer Funktionen“. Die Schwierigkeit im Umgang mit FVG bestehe nunmehr lediglich darin, „die im Einzelfall relevante Funktion zu ermitteln“ (ebd.). Damit erhebt sich die Frage, ob angesichts der Vielzahl potentieller Funktionen, die FVG offenbar nicht nur in grammatischer, lexikalischer und stilistisch-pragmatischer Hinsicht tragen können, überhaupt noch von einer zusammengehörigen Klasse von FVG die Rede sein kann. Eine Bestimmung der FVG von einer Funktion bzw. von einem begrenzten Set von Funktionen her, wie sie von Polenz vornimmt, ist angesichts dieser funktionellen Vielfalt jedenfalls kaum mehr möglich. Dass Nomen-Verb-Verbindungen Funktionen besitzen, ist schließlich ein trivialer Sachverhalt: Syntaktische Verbindungen, die nicht auf irgendeiner Ebene der Sprache eine ihnen vom Sprecher zugedachte semantische oder pragmatische Funktion erfüllen, sind kaum denkbar.

Die Schwierigkeiten, die durch die Auffächerung des bei von Polenz noch relativ konsistenten Funktionsbegriffs in ein ganzes Spektrum unterschiedlichster Funktionen für die Begründung einer Klasse FVG entstehen, hat die Forschung zumindest im Ansatz erkannt und auf unterschiedliche Weise zu beheben versucht. So gesteht Helbig (1984: 175) zwar zu, „daß die Anwendung aller Kriterien kein einheitliches Bild ergibt, daß sich die einzelnen FVG bei der Anwendung der genannten Kriterien unterschiedlich verhalten“. Diese Situation wird jedoch nicht als problematisch gewertet. Vielmehr wird die Forderung nach absolut geltenden Kriterien und damit nach einer homogenen Klasse von FVG als überzogen zurückgewiesen. Eine solche Forderung, die einen „Primat der Methode über den Gegenstandsbereich“ beinhalte, führe „zu einer für die Sprachwissenschaft nicht zu rechtfertigenden Einschränkung ihres Gegenstandsbereiches, zum ,strukturalistischen Reduktionismus““ (ebd.). Helbig selbst vertritt demgegenüber einen „Primat des Gegenstandes“: Der sprachliche Gegenstand lasse sich mit linguistischen Methoden nicht vollständig und systematisch abbilden, relative Kriterien und inhomogene Kategorien seien vor diesem Hintergrund in Kauf zu nehmen. Dieser von Helbig favorisierte Ausweg aus dem Beschreibungsdilemma ist jedoch nicht akzeptabel, da ein Primat des Gegenstandes vor der Methode wissenschaftstheoretisch naiv ist. ${ }^{44}$ Wissenschaftliche Gegenstände sind nicht a priori

44 Explizit auf den „Primat des Gegenstandes“ und die „Sprachrealität“ beruft sich übrigens auch Kamber (2008: 15, 43) in seiner strikt korpusbezogenen Untersuchung zu FVG (allerdings ohne Klärung seines Verhältnisses zur Wiederspiegelungstheorie Helbigs). Dementsprechend 
gegeben, sondern konstituieren sich erst als Produkte eines methodengeleiteten Erkenntnisprozesses. Wie im Fall der FVG zu beobachten, kann allenfalls die sukzessive Verfestigung von Forschungstraditionen zu dem Trugschluss führen, es lägen tatsächlich fertige Gegenstände vor, die in einer linguistischen Beschreibung dann nur noch zu spiegeln wären. ${ }^{45}$

Das Scheitern der bisherigen Abgrenzungsversuche wird in der neueren Literatur - vor allem im Gefolge des kritischen Buchs von van Pottelberge (2001) vielfach mehr oder weniger ausdrücklich eingeräumt (vgl. Seifert 2004: 12f.; Fabricius-Hansen 2005: 425; Eisenberg 2006b: 299; Heine 2006: 75; Helbig 2006: 167; Kamber 2008: 11f.). Im Fehlen einer klaren Definition bzw. eines klar bestimmbaren Sets von klassenkonstituierenden Merkmalen wird allgemein jedoch kein Grund gesehen, die Kategorie FVG grundsätzlich zur Disposition zu stellen. Diesem Mangel versuchen nicht wenige Forscher durch die Annahme einer prototypischen Organisation der Klasse FVG zu begegnen. So heißt es etwa bei Seifert (2004: 54), dass ${ }^{46}$

die [...] Merkmale [von FVG, V. H.] nicht im Sinne einer starren Ausschließlichkeit gemeint [sind], sondern sie charakterisieren den jeweiligen Prototyp. Mit Ausnahmen und atypi-

hält Kamber (2008: 1) die Definitionsproblematik bei FVG für überwertet und möchte stattdessen die „Fakten“ beschreiben. Wie sich sprachliche „Fakten“ konstituieren, wird nicht reflektiert.

45 Helbig (1984: 175) beruft sich in der Tat explizit auf die marxistische Widerspiegelungstheorie. Vor diesem ideologischen Hintergrund ist auch der Vorwurf des „strukturalistischen Reduktionismus“ zu verstehen: Der Strukturalismus wurde in der Sprachwissenschaft der DDR u. a. wegen „unwissenschaftlicher philosophischer Positionen“ (Motsch 1974: 56, hier zitiert nach Helbig 1986: 68) kritisiert, zu denen auch die „undialektische Verabsolutierung der Methoden vom Gegenstand der Sprachwissenschaft“ gerechnet wurde (Motsch 1974: 104; hier nach Helbig 1986: 68f.). - Helbigs Polemik erinnert zudem an den in der Epoche des amerikanischen Strukturalismus ausgetragenen Methodenstreit, in dem eine faktenorientierte „god's truth“-Linguistik und eine modelltheoretische ausgerichtete ,Hokuspokus-Linguistik ' einander gegenübergestellt wurden.

46 Seifert (2004: 53, Fußnote 2) weist mit Recht darauf hin, dass die Annahme einer prototypischen Kategorienstruktur sich - zumindest implizit - bereits in älteren Arbeiten findet, wenn dort von „Zentrum“ und „Peripherie“ o. ä. die Rede ist, vgl. neben den bei Seifert genannten Autoren auch Heringer (1968: 53f.), bei dem ein ,innerer und äußerer Bereich von Funktionsverfügungen " unterschieden wird. 
schen Beispielen ist durchaus zu rechnen, weil die Klassifikation nicht auf der Basis gemeinsamer Eigenschaften im Sinne hinreichender und notwendiger Bedingungen beruht, sondern sich auf einen Vergleich mit dem Prototyp stützt. ${ }^{47}$

Ob eine prototypentheoretische Herangehensweise an sprachwissenschaftliche Kategorien grundsätzlich sinnvoll ist, kann an dieser Stelle nicht diskutiert werden. Immerhin wäre $\mathrm{zu}$ fragen, ob wissenschaftliche Kategorienbildung sich nicht wesentlich von den „folk taxonomies“ unterscheidet (oder unterscheide sollte), die den eigentlichen Gegenstand der Prototypensemantik bilden. ${ }^{48}$ Unterzieht man, unabhängig von solch grundsätzlichen Fragen, Seiferts Anwendung der Prototypentheorie auf FVG einer näheren Betrachtung, wird deutlich, dass auch hiermit keine befriedigende Lösung des Abgrenzungsproblems erreicht ist. So sucht man vergeblich nach einer methodisch abgesicherten Aussage darüber, was der Prototyp der Kategorie FVG denn eigentlich sei. Seifert bemüht zur Bestimmung des Prototyps lediglich die angebliche communis opinio der Forschung:

Den Kernbereich der FVG bilden nach allgemeiner Auffassung die Konstruktionen des Typs zur Anwendung bringen. Diese FVG haben die Forschung am meisten fasziniert und sollen auch in der vorliegenden Arbeit im Mittelpunkt stehen.

(Seifert 2004: 54)

Auch Eisenberg (2006a: 310) zieht zur Eingrenzung eines Kernbereichs von FVG lediglich ein Autoritätsargument heran: „Den Kernbereich der FVG bilden nach fast einhelliger Meinung in der Literatur Ausdrücke aus FV + PrGr [Präpositionalgruppe, V. H.]“. Wenn man tatsächlich eine prototypische Strukturierung der Klasse der FVG annimmt, bedürfte es anstelle der Berufung auf eine vage ,allgemeine Auffassung“ bzw. „einhellige Meinung“ wissenschaftlich relevanter und überprüfbarer Kriterien zur Bestimmung des Kategorienzentrums. Welche Kriterien dies sein könnten, ist allerdings gegenwärtig vollkommen unklar.

Festzuhalten bleibt also, dass auch die jüngere und jüngste Forschung keine weiterführenden Ansätze zur Lösung des Abgrenzungsproblems bietet. Der An-

47 Explizit auf die Prototypensemantik beruft sich Helbig auch in seiner jüngsten Auseinandersetzung mit dem sich bei FVG stellenden Klassifikationsproblem (2006: 167f.); für eine prototypische Struktur der Kategorie ,FVG‘ plädieren auch Fabricius-Hansen (2005: 425), Eisenberg (2006a: 310), Heine (2006: 48) und Kamber (2008: 20).

$48 \mathrm{Zu}$ unterschiedlichen Strukturprinzipien von „folk categories“ und „expert categories“ vgl. Taylor (1995: 68-74). 
satz einer prototypischen Kategorie FVG mit einem - wie auch immer zu bestimmenden -Zentrum und einer Peripherie hat den Charakter einer Verlegenheitslösung. Prototypen sind auch hier ,an excuse for not ever defining anything“, wie Wierzbicka (1996: 160) einmal in anderem Zusammenhang festgehalten hatte.

Angesichts der Situation, dass die bisher vorgestellten Abgrenzungskriterien sich als zu unspezifisch und daher als unbrauchbar erwiesen haben, stellen sich zwei Alternativen: Die eine Möglichkeit besteht darin, die Kategorie FVG, wie van Pottelberge (2001) vorschlägt, grundsätzlich aufzugeben oder bestenfalls als intuitive Charakterisierung gelten zu lassen. Eine andere Möglichkeit aber wäre die, das Problem der Nomen-Verb-Verbindungen von einer anderen Seite her anzugehen, als bisher üblich war, und so möglicherweise doch zu einer konsistenten Klassenbildung zu gelangen. Dieser letzte Weg soll in der vorliegenden Untersuchung beschritten werden. Ansatzpunkt der erneuten Betrachtung von Nomen-Verb-Verbindungen soll dabei nicht der bisher zentrale Aspekt - die Frage nach systematisch beschreibbaren Funktionen wie Aktionsart, Kausativ, Passiv - sein. Es soll auch nicht, wie seit von Polenz (1963) durchgängig der Fall, vom Verb als dem Kern der Fügung ausgegangen werden. Im Mittelpunkt des Definitionsversuches, der in dem folgenden Kapitel vorgelegt wird, steht vielmehr die Klassifikation der Verbindung zwischen Nomen und Verb. FVG sollen daher als Konstruktionstypus beschrieben werden, der sich von anderen Konstruktionen in signifikanter Weise unterscheidet. 